\title{
Smoothness-Increasing Accuracy-Conserving (SIAC) Filtering for Discontinuous Galerkin Solutions over Nonuniform Meshes: Superconvergence and Optimal Accuracy
}

\author{
Xiaozhou $\mathrm{Li}^{1}$ • Jennifer K. Ryan ${ }^{2,3} \cdot$ Robert M. Kirby ${ }^{4}$ Kees Vuik ${ }^{5}$
}

Received: 23 August 2018 / Revised: 23 January 2019 / Accepted: 29 January 2019 /

Published online: 13 February 2019

(C) The Author(s) 2019

\begin{abstract}
Smoothness-increasing accuracy-conserving (SIAC) filtering is an area of increasing interest because it can extract the "hidden accuracy" in discontinuous Galerkin (DG) solutions. It has been shown that by applying a SIAC filter to a DG solution, the accuracy order of the DG solution improves from order $k+1$ to order $2 k+1$ for linear hyperbolic equations over uniform meshes. However, applying a SIAC filter over nonuniform meshes is difficult, and the quality of filtered solutions is usually unsatisfactory applied to approximations defined on nonuniform meshes. The applicability to such approximations over nonuniform meshes is the biggest obstacle to the development of a SIAC filter. The purpose of this paper is twofold: to study the connection between the error of the filtered solution and the nonuniform mesh and to develop a filter scaling that approximates the optimal error reduction. First, through analyzing the error estimates for SIAC filtering, we computationally establish for the first time a relation between the filtered solutions and the unstructuredness of nonuniform meshes. Further, we demonstrate that there exists an optimal accuracy of the filtered solution for a given nonuniform mesh and that it is possible to obtain this optimal accuracy by the method we propose, an optimal filter scaling. By applying the newly designed filter scaling over nonuniform meshes, the filtered solution has demonstrated improvement in accuracy order as well as reducing the error compared to the original DG solution. Finally, we apply the proposed methods over a large number of nonuniform meshes and compare the performance with existing methods to demonstrate the superiority of our method.
\end{abstract}

Keywords Discontinuous Galerkin method · Post-processing · SIAC filtering · Superconvergence $\cdot$ Nonuniform meshes $\cdot$ Unstructuredness

In memory of Saul Arbarbenel, a dear friend and mentor.

Xiaozhou Li: Supported by the National Natural Science Foundation of China (NSFC) under Grants No. 11801062 and the Fundamental Research Funds for the Central Universities. Xiaozhou Li and Jennifer K. Ryan: Supported by the Air Force Office of Scientific Research (AFOSR), Air Force Material Command, USAF, under Grant number FA8655-13-1-3017. Robert M. Kirby: Supported by the Air Force Office of Scientific Research (AFOSR), Computational Mathematics Program (Program Manager: Dr. Fariba Fahroo), under Grant Number FA9550-12-1-0428.

Extended author information available on the last page of the article 


\section{Introduction}

In practical applications, there are strong motivators for the adoption of unstructured meshes for handling complex geometries and using adaptive mesh refinement techniques. Based on this practical necessity, it is widely believed that discontinuous Galerkin methods, which provide high-order accuracy on unstructured meshes, will become one of the standard numerical methods for future generations. Along with the rapid growth of the DG method, the superconvergence of the DG method has become an area of increasing interest because of the ease with which higher order information can be extracted from DG solutions by applying smoothness-increasing and accuracy-conserving (SIAC) filtering. However, SIAC filters are still limited primarily to structured meshes. For general nonuniform meshes, the quality of the filtered solution is usually unsatisfactory. The ability to effectively handle nonuniform meshes is an obstacle to the further development of a SIAC filter.

This paper focuses on applying a SIAC filter for DG solutions over nonuniform meshes. Specifically, this study focuses on the barrier to applying SIAC filters over nonuniform meshes - the scaling. This problem was noted in [3], which extends a postprocessing technique for enhancing the accuracy of solutions [1] to linear hyperbolic equations. The postprocessing technique was renamed the Smoothness-Increasing Accuracy-Conserving filter in [5]. A series of studies of different aspects of SIAC filters are presented in $[5,11,20]$. For uniform meshes, it was shown that by applying a SIAC filter to a DG approximation at the final time, the accuracy order improves from $k+1$ to $2 k+1$ for linear hyperbolic equations with periodic boundary conditions [3]. This superconvergence of order $2 k+1$ is promising; however it is limited to uniform meshes. Only for a particular family of nonuniform meshes, smoothly-varying meshes, have the filtered solutions been proven to have a superconvergence order of $2 k+1$ [20]. As for general nonuniform meshes, the preliminary theorem in [3] provides a solution, but it is not very useful in practice. The filtered solutions can still be improved. Further, the computational results for relatively unstructured triangular meshes [12] suggest that it is possible to reduce the errors of the DG solutions through a suitable choice of filter scalings for approximations defined over unstructured meshes. However, in [12] there is no clear accuracy order improvement and no guarantee of error reduction. Also, the lack of theoretical analysis makes it difficult to evaluate the quality of the filtered solutions. There has been some work related this topic, such as the nonuniform filter proposed in $[15,16]$.

The primary goal of this paper is to address these challenges and try to improve the quality of the DG solutions over general nonuniform meshes. Our main contributions are:

Optimal accuracy First, we study the error estimates of the SIAC filter for uniform and nonuniform meshes and point out the difficulties for the filter over nonuniform meshes. Then, we computationally establish for the first time a relation between the filtered solutions and the unstructuredness of nonuniform meshes. Further, we demonstrate that for a given nonuniform mesh, there exists an optimal accuracy (optimal error reduction) of the filtered solution.

Optimal scaling To approximate this optimal accuracy, we first analyze the relation between the filter scaling and the error of filtered solutions for different nonuniform meshes. Then, we introduce a measure of the unstructuredness of nonuniform meshes and propose a procedure that adjusts the scaling of a SIAC filter according to the unstructuredness of the given nonuniform mesh. Also, we demonstrate that with the newly designed optimal scaling, the filtered solution has a higher accuracy order, and the errors are reduced compared to the original DG solutions even for the worst nonuniform meshes. 
Scaling performance validation Finally, to ensure the proposed scaling is a robust algorithm that can be used in practice, we validated the performance of the proposed scaling over a large number of nonuniform meshes and compared with other commonly used scalings to illustrate that the accuracy of the DG solution is improved by using the proposed scaling and its superiority compared to existing methods.

This paper is organized as follows. In Sect. 2, we review the DG method and SIAC filters as well as the relevant properties. In Sect. 3, we investigate the effects of the filter scaling on the accuracy of the filtered solution. We then introduce a measure of the unstructuredness of nonuniform meshes and provide an algorithm to approach the optimal accuracy in Sect. 4. Also, in Sect. 4, we provide a scaling performance validation for the proposed scaling along with other commonly used scalings. Numerical results for different one- and two-dimensional nonuniform meshes are given in Sect. 5. The conclusions are presented in Sect. 6.

\section{Background}

In this section, we review the necessary properties of discontinuous Galerkin methods, the definition of nonuniform meshes for the purposes of this article, and the Smoothness-Increasing Accuracy-Conserving (SIAC) filter.

\subsection{Construction of Nonuniform Meshes}

Before introducing the discontinuous Galerkin method, we introduce the structure of the nonuniform meshes that will be used in this paper. The main construction of the nonuniform meshes are similar to those meshes used in [11]:

\section{Mesh 2.1}

$$
x_{\frac{1}{2}}=0, \quad x_{N+\frac{1}{2}}=1, \quad x_{j+\frac{1}{2}}=\left(j+b \cdot r_{j+\frac{1}{2}}\right) h, \quad j=1, \ldots, N-1
$$

where $\left\{r_{j+\frac{1}{2}}\right\}_{j=1}^{N-1}$ are random numbers between $(-1,1)$, and $b \in(0,0.5]$ is a constant number. Here, $h=\frac{x_{N+\frac{1}{2}}-x_{\frac{1}{2}}}{N}$ is a function of $N$, in this way, one can reduce the structure added by increasing the number of elements. The size of element $\Delta x_{j}=x_{j+\frac{1}{2}}-x_{j-\frac{1}{2}}$ is between $((1-2 b) h,(1+2 b) h)$. In order to save space, we present an example with $b=0.4$ only. Other values of $b$ such as 0.1, 0.2 and 0.45 have also been studied and are consistent with the results presented herein.

Mesh 2.2 We distribute the element interface, $x_{j+\frac{1}{2}}, j=1, \ldots, N-1$, randomly for the entire domain and only require

$$
\Delta x_{j}=x_{j+\frac{1}{2}}-x_{j-\frac{1}{2}} \geq b \cdot h, \quad j=0, \ldots, N .
$$

In this paper (except the performance tests in Sect. 4), we present the case where $b=0.5$ for this mesh. Other values of $b$ such as 0.6, 0.8 have also been studied and are consistent with the results presented herein.

Remark 2.3 Mesh 2.1 is a quasi-uniform mesh since $\frac{\Delta x_{\max }}{\Delta x_{\min }} \leq \frac{1+2 b}{1-2 b}$. Mesh 2.2 is more unstructured than Mesh 2.1 since in the worst case $\frac{\Delta x_{\max }}{\Delta x_{\min }} \approx \frac{1-b}{b} N$ which is unbounded as $N \rightarrow \infty$. 
Mesh 2.1

Mesh 2.2

Fig. 1 Illustration of Meshs 2.1 and 2.2. Here the largest-to-smallest element ratio is about 4.5 for Mesh 2.1 (top), and 33.1 for Mesh 2.2 (bottom)

It is expected that the DG approximation and the filtered solution are of better quality for Mesh 2.1 than for Mesh 2.2. Illustrations of these meshes are given in Fig. 1.

We will analyze the applicability of the SIAC filter scaling factor utilizing these meshes.

\subsection{Discontinuous Galerkin Methods}

Here, we briefly describe the discontinuous Galerkin method; more details can be found in $[2,4]$. As an illustrative example, we consider a multi-dimensional linear hyperbolic equation of the form

$$
\begin{aligned}
u_{t}+\sum_{i=1}^{d} A_{i} u_{x_{i}}+A_{0} u & =0, \quad(\mathbf{x}, t) \in \Omega \times[0, T], \\
u(\mathbf{x}, 0) & =u_{0}(\mathbf{x}),
\end{aligned}
$$

where $u_{0}$ is sufficiently smooth, the coefficients $A_{i}$ are constants and $\Omega=\left[a_{1}, b_{1}\right] \times \cdots \times$ $\left[a_{d}, b_{d}\right] \subset \mathbb{R}^{d}$. Let $K$ represent an element in a quadrilateral tessellation $\mathcal{T}_{h}$ of the domain $\Omega$. Discontinuous Galerkin methods seek an approximation $u_{h}$ in the space of piecewise polynomials of degree $\leq k$,

$$
V_{h}^{k}=\left\{\varphi:\left.\varphi\right|_{K} \in \mathbb{P}^{k}, \quad \forall K \in \mathcal{T}_{h}\right\},
$$

and the DG approximation $u_{h}$ is determined by the scheme

$$
\left(\left(u_{h}\right)_{t}, v_{h}\right)_{K}-\sum_{i=1}^{d}\left(a_{i} u_{h},\left(v_{h}\right)_{x_{i}}\right)_{K}+\sum_{i=1}^{d} \int_{\partial K} a_{i} \hat{u}_{h} v_{h} n_{i} d s+\left(a_{0} u_{h}, v_{h}\right)_{K}=0
$$

for any $v_{h} \in V_{h}^{k}$, and $\hat{u}_{h}$ is the flux. For the results presented in this paper, we have utilized one particular choice- the upwind flux. Here, $(f, g)$ denotes the standard inner product:

$$
(f, g)_{K}=\int_{K} f g d K \text {. }
$$

\subsection{Superconvergence in the Negative Order Norm}

The DG method has many important properties. The most relevant property for the purposes of this paper are the accuracy order of the divided differences of the DG approximation. In the $L^{2}$ norm it is $k+1$ which aides in proving the superconvergence of order $2 k+1$ in the negative order norm. These properties are the theoretical foundations of SIAC filters (see $[3,11]$ ) and define the choice of the number of B-splines in the SIAC convolution kernel. To highlight this connection, the error of filtered solution can be viewed a linear combination 
of the errors from the choice of the number of B-splines used in the filter as well as the discretization error,

$$
\left\|u-u_{h}\right\|_{0} \leq \underbrace{C_{1} H^{2 k+1}}_{\text {Number of B-Splines }}+C_{2} \underbrace{\left\|\partial_{H}^{\alpha}\left(u-u_{h}\right)\right\|_{-(k+1)}}_{\text {Discretization Error }} .
$$

This is discussed further in Sect. 3.2. Because of the importance of the divided differences in the error estimates, in this section, we first discuss the properties of the divided difference of DG approximation. For uniform meshes, the main theorem is given below.

Theorem 2.1 (Cockburn et al. [3]) Let $u$ be the exact solution of Eq. (2.1) with periodic boundary conditions, and $u_{h}$ the DG approximation derived by scheme (2.2). For a uniform mesh, the approximation and its divided differences in the $L^{2}$ norm are:

$$
\left\|\partial_{h}^{\alpha}\left(u-u_{h}\right)\right\|_{0, \Omega} \leq C h^{k+1}
$$

and in the negative order norm:

$$
\left\|\partial_{h}^{\alpha}\left(u-u_{h}\right)\right\|_{-(k+1), \Omega} \leq C h^{2 k+1},
$$

where $\alpha=\left(\alpha_{1}, \ldots, \alpha_{d}\right)$ is an arbitrary multi-index and $h$ is the diameter of the uniform elements.

This theorem is valid assuming that the exact solution has sufficient regularity (belongs to a Hilbert space of order $2 k+2$ ). Unfortunately, the error estimates of the DG approximation and its divided differences for nonuniform meshes become much more challenging, and for this case the estimates (2.3) and (2.4) are valid only for the DG approximation itself, that is,

Lemma 2.2 (Cockburn et al. [3]) Under the same conditions as in Theorem 2.1. The DG approximation for a nonuniform mesh satisfies

$$
\left\|u-u_{h}\right\|_{0, \Omega} \leq C h^{k+1},
$$

and in the negative order norm:

$$
\left\|u-u_{h}\right\|_{-(k+1), \Omega} \leq C h^{2 k+1} .
$$

For the divided differences, $\partial_{h}^{\alpha} u_{h}$, for nonuniform meshes, instead of (2.4), we have only the following lemma:

Lemma 2.3 Under the same conditions as in Lemma 2.2, given a constant scaling $H$, for nonuniform meshes, the divided differences of the DG approximation in the $L^{2}$ norm satisfies

$$
\left\|\partial_{H}^{\alpha}\left(u-u_{h}\right)\right\|_{0, \Omega} \leq C_{\alpha} h^{2 k+1} H^{-|\alpha|},
$$

and in the negative order norm:

$$
\left\|\partial_{H}^{\alpha}\left(u-u_{h}\right)\right\|_{-(k+1), \Omega} \leq C_{\alpha} h^{2 k+1} H^{-|\alpha|},
$$

where $\alpha=\left(\alpha_{1}, \ldots, \alpha_{d}\right)$ is an arbitrary multi-index.

Proof c.f. $[11,14]$.

Remark 2.4 Lemma-2.3 was first introduced as a conjecture in [3], and presented as a lemma with proof in [11]. In this paper, $h$ is defined during the construction of Meshs 2.1 and 2.2, $h=\frac{x_{N+\frac{1}{2}}-x_{1}}{N}$ is a function of the element $N$. Here, we note that Lemma 2.3 is valid for arbitrary constant $H$, but we will discuss how to choose the optimal scaling $H$ in the following sections. 
The relation between the $L^{2}$ norm and the negative order norms are introduced in the following lemma:

Lemma 2.4 (Bramble and Schatz [1]) Let $\Omega_{0} \subset \subset \Omega_{1}$ and $s$ be an arbitrary but fixed nonnegative integer. Then for $u \in H^{s}\left(\Omega_{1}\right)$, there exists a constant $C$ such that

$$
\|u\|_{0, \Omega_{0}} \leq C \sum_{|\alpha| \leq s}\left\|D^{\alpha} u\right\|_{-s, \Omega_{1}} .
$$

In Table 1, we provide a basic example of the divided difference operation over a nonuniform mesh (randomly chosen among Meshes 2.2). In this table, $\mathcal{P} u$ is the $L^{2}$ projection of $u(x, 0)=\sin (x)$ over a randomly generated nonuniform mesh. From Table 1 , we can see that for $\alpha \geq 1$, the divided differences $\partial_{h}^{\alpha} \mathcal{P} u$ only have accuracy order of $k+1-\alpha$. This example clearly suggests that the nonuniform mesh estimate (2.5) no longer holds, and the estimates in Lemma 2.3 can not be improved without further assumptions on the nonuniformity of the mesh.

Remark 2.5 In this paper, the main results are based on the $L^{2}$ norm. However, we also included the numerical results in the $L^{\infty}$ norm for consistency with existing literature.

\subsection{SIAC Filter}

We use the classical SIAC filter that stems from the work of Bramble and Schatz [1], Thomée [22] and Mock and Lax [14]. An extension of this technique to discontinuous Galerkin methods was introduced in [3]. Motivated by [3], a series of publications have studied SIAC filtering for DG methods from various aspects, such as [5,12,18,19,21].

SIAC filtering is applied only at the final time $T$ of the DG approximation, and the filtered solution $u_{h}^{\star}$, in the one-dimensional case is given by

$$
u_{h}^{\star}(x, T)=\left(K_{H}^{(2 r+1, \ell)} \star u_{h}\right)(x, T)=\int_{-\infty}^{\infty} K_{H}^{(2 r+1, \ell)}(x-\xi) u_{h}(\xi, T) d \xi,
$$

where the filter, $K^{(2 r+1, \ell)}$, is a linear combination of central B-splines,

$$
K^{(2 r+1, \ell)}(x)=\sum_{\gamma=0}^{2 r} c_{\gamma}^{(2 r+1, \ell)} \psi^{(\ell)}\left(x-\left(-\frac{r}{2}+\gamma\right)\right),
$$

and the scaled filter is $K_{H}^{(2 r+1, \ell)}(x)=\frac{1}{H} K^{(2 r+1, \ell)}\left(\frac{x}{H}\right)$ with scaling $H$ ( $H=h$ for uniform meshes). Here, $\psi^{(\ell)}(x)$ is the $\ell$ order central B-spline, which can be constructed recursively using the relation

$$
\begin{aligned}
\psi^{(1)}= & \chi_{[-1 / 2,1 / 2)}(x), \\
\psi^{(\ell)}(x)= & \frac{1}{\ell-1}\left(\frac{\ell}{2}+x\right) \psi^{(\ell-1)}\left(x+\frac{1}{2}\right) \\
& +\frac{1}{\ell-1}\left(\frac{\ell}{2}-x\right) \psi^{(\ell-1)}\left(x-\frac{1}{2}\right), \quad \ell \geq 2 .
\end{aligned}
$$

Typically, the number of B-splines is chosen as $2 r+1=2 k+1$, and the order of B-splines is chosen as $\ell=k+1$. In the remainder of the paper, we use $2 k+1 \mathrm{~B}$-splines of order 


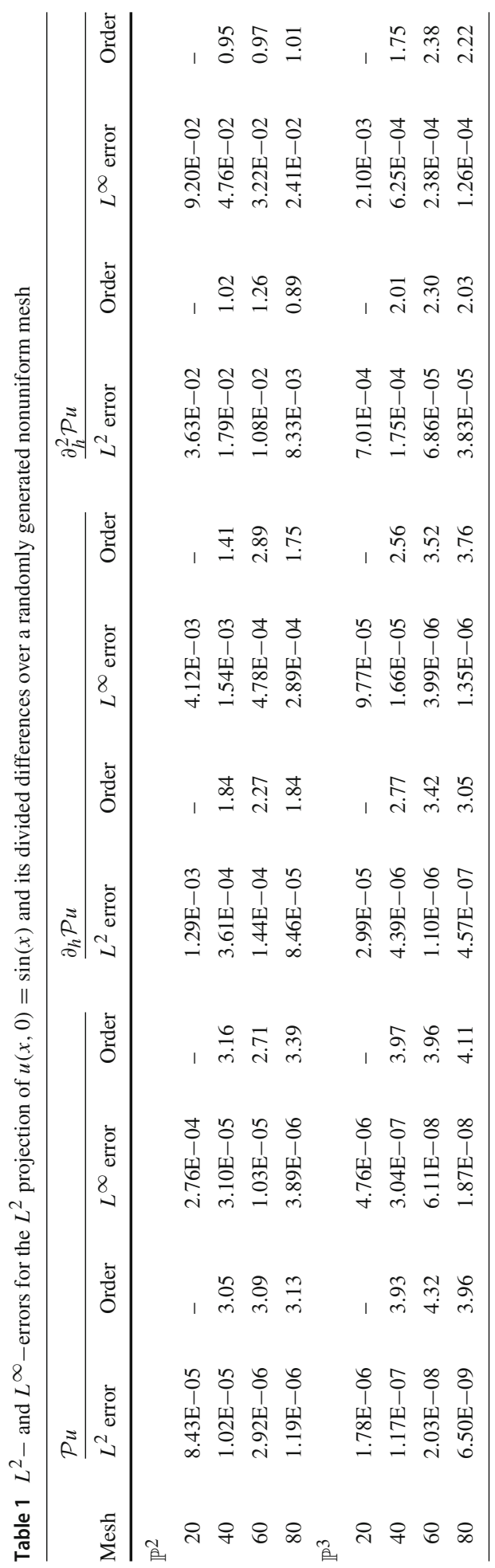


$k+1$. The coefficients, $c_{\gamma}^{(2 r+1, \ell)}$, are calculated by enforcement of the property that the filter reproduces polynomials by convolution up to degree $2 r$,

$$
K^{(2 r+1, \ell)} \star p=p, \quad p=1, x, \ldots, x^{2 r} .
$$

Later on we will need the following lemma

Lemma 2.5 Let $2 r$ be an even number, then the SIAC filter $K^{(2 r+1, \ell)}$ given in (2.6), which satisfies (2.8), reproduces polynomials by convolution until degree of $2 r+1$,

$$
K^{(2 r+1, \ell)} \star p=p, \quad p=1, x, \ldots, x^{2 r+1} .
$$

Proof c.f. [23].

In the multidimensional case, the multidimensional filter is the tensor product of the one-dimensional filter (2.6)

$$
\mathbf{K}_{H}^{(2 r+1, \ell)}(\mathbf{x})=\prod_{i=1}^{d} K_{H}^{(2 r+1, \ell)}\left(x_{i}\right), \quad \mathbf{x}=\left(x_{1}, \ldots, x_{d}\right) \in \mathbb{R}^{d},
$$

with the scaled filter $\mathbf{K}_{H}^{(2 r+1, \ell)}(\mathbf{x})=\frac{1}{H^{d}} K^{(2 r+1, \ell)}\left(\frac{\mathbf{x}}{H}\right)$. A computationally efficient alternative to the tensor product case is to use the Hexagonal SIAC filter (HSIAC) by Mirzarger et al. [13], or the Line SIAC filter introduced by Docampo et al. [6] and applied to problems in visualization problems by Jallepalli et al. [9].

\section{SIAC Filter for Nonuniform Meshes}

In order to design a more accurate SIAC filter for nonuniform meshes, we have to investigate the relations between the DG approximation and SIAC filters for nonuniform meshes.

\subsection{Existing Results}

As mentioned in $[3,10]$, for uniform meshes, SIAC filtering can improve the accuracy order of DG solutions for linear hyperbolic equations from $k+1$ to $2 k+1$ when a sufficient number of B-splines are chosen. This accuracy order, $2 k+1$, and various studies of SIAC filters, such as position-dependent filters [19,24], the derivative filter [18], etc., are limited to uniform meshes. For nonuniform meshes, the aims of improving the accuracy order and reducing the errors of the DG solution remains an ongoing challenge for SIAC filtering. Most preliminary results consider only a particular family of meshes, smoothly varying meshes $[5,17,20]$. It was proven in [20] that the filtered solutions also have an accuracy order of $2 k+1$ for smoothly varying meshes. However, for general nonuniform meshes, there are only a few computational results [12], and the only theoretical estimates were given in [3,11].

Theorem 3.1 Under the same conditions as in Lemma 2.2, denote $\Omega_{0}+2 \operatorname{supp}\left(K_{H}^{(2 k+1, k+1)}\right)$ $\subset \subset \Omega_{1} \subset \subset \Omega$. Then, for general nonuniform meshes, we have

$$
\left\|u-K_{H}^{(2 k+1, k+1)} \star u_{h}\right\|_{0, \Omega_{0}} \leq C h^{\mu(2 k+1)},
$$

where the scaling $H$ is chosen as

$$
H=h^{\mu}, \quad \mu=\frac{2 k+1}{3 k+2} .
$$


Proof c.f. $[3,11]$.

For convenience, in this paper we refer to $\mu$ as the scaling order and $\mu_{0}=\frac{2 k+1}{3 k+2}$. Theorem 3.1 gives a useful scaling that allows us to enhance the accuracy of the DG solution, especially the derivatives of the DG solution [11], but may not be optimal.

However, from the perspective of improving the DG approximation itself, satisfying the requirements of Theorem 3.1 can be cumbersome. For example, the accuracy order will be higher than the original DG approximation only if $k \geq 2$ :

$$
\mu_{0}(2 k+1)>k+1 \Rightarrow k \geq 2(k \in \mathbb{Z}) .
$$

If, alternatively, at least one order higher accuracy order is desired, then $k \geq 5$ :

$$
\mu_{0}(2 k+1) \geq k+2 \Rightarrow k \geq 5(k \in \mathbb{Z}) .
$$

Another important issue is the computational efficiency. As discussed in [11] when $h$ is small (a fine mesh), the filter scaling $H=h^{\mu_{0}} \geq h^{2 / 3}$ dramatically increases the support size of the filter. To post-process one position in the domain, the post-processor has a support of $(3 k+2) H$. It follows that by choosing $\mu<1$, the computational cost dramatically increases.

More importantly, instead of increasing the accuracy order, practical applications are more concerned with reducing the error. Although using the scaling $H=h^{\mu_{0}}$ improves the accuracy order, many practical examples suggest that using a scaling order of $\mu_{0}$ usually increases the errors. For example, for the numerical experiments given in this paper (Sect. 5), the filtered solutions that use a scaling order of $\mu_{0}$ have a qualitatively worse error in the $L^{2}$ norm compared to the original DG solutions.

\subsection{The Optimal Accuracy}

Although Theorem 3.1 holds for arbitrary nonuniform meshes, the filtered solutions based on the filter scaling $H=h^{\mu_{0}}$ does not achieve expectations with respect to order improvement, error reduction and computational efficiency. The problem stems from the crude estimate of the scaling order $\mu_{0}$ that ignores the mesh structure. In order to improve Theorem 3.1 , it is necessary to reconsider the filter scaling for nonuniform meshes. To complete this task, we first explore the relation between the filter scaling and the error of the filtered solution. We remind the reader that in this paper, $H$ represents the filter scaling and $h$ represents the mesh size. As given in [3], we can write the error estimate of the filtered solution as

$$
\left\|u-u_{h}^{\star}\right\|_{0, \Omega_{0}} \leq \Theta_{1}+\Theta_{2},
$$

where

$$
\Theta_{1}=\left\|u-K_{H}^{(2 k+1, k+1)} \star u\right\|_{0, \Omega_{0}} \leq C_{1} H^{2 k+2}|u|_{H^{2 k+2}},
$$

and

$$
\begin{aligned}
\Theta_{2} & =C_{0} \sum_{|\alpha| \leq k+1}\left\|D^{\alpha} K_{H}^{(2 k+1, k+1)} \star\left(u-u_{h}\right)\right\|_{-(k+1), \Omega_{1 / 2}} \\
& \leq C_{0} C_{1} \sum_{|\alpha| \leq k+1}\left\|\partial_{H}^{\alpha}\left(u-u_{h}\right)\right\|_{-(k+1) \Omega_{1}},
\end{aligned}
$$

by Lemmas 2.5 and 2.4, where

$$
\Omega_{0}+\operatorname{supp}\left(K_{H}^{(2 k+1, k+1)}\right) \subset \Omega_{1 / 2}, \quad \Omega_{1 / 2}+\operatorname{supp}\left(K_{H}^{(2 k+1, k+1)}\right) \subset \Omega_{1} .
$$


According to the above estimates, the error is bounded by $\Theta_{1}$ and $\Theta_{2}$, where $\Theta_{1}$ describes the error generated by reproducing polynomials and $\Theta_{2}$ represents the error in the negative order norm.

The estimate for $\Theta_{1}$ is clear. The error is given by the polynomial reproduction property (2.9) and the exact solution $u$. It is obvious from (3.3) that $\Theta_{1}$, only depends on the filter scaling and is bounded by $C_{1} H^{2 k+2}|u|_{H^{2 k+2}}$. This bound increases with the scaling $H$.

The $\Theta_{2}$ term is more challenging. Lemma 2.3 gives an estimate of $\left\|\partial_{H}^{\alpha}\left(u-u_{h}\right)\right\|_{-(k+1), \Omega_{1}}$ for nonuniform meshes,

$$
\left\|\partial_{H}^{\alpha}\left(u-u_{h}\right)\right\|_{-(k+1), \Omega_{1}} \leq C h^{2 k+1} H^{-|\alpha|} .
$$

The above estimate holds for arbitrary nonuniform meshes, but it is not the optimal bound for many meshes. For example, consider the smoothly-varying meshes used in $[5,11,20]$. For these types of meshes, a better estimate is

$$
\left\|\partial_{H}^{\alpha}\left(u-u_{h}\right)\right\|_{-(k+1), \Omega_{1}} \leq C h^{2 k+1}
$$

for well chosen $H$, see [20]. Clearly, one can guess that the accurate bounds of $\Theta_{2}$ are very different between an almost uniform mesh and a totally random mesh, but the current estimate (3.5) fails to relize this relation (the relation between $\Theta_{2}$ and the unstructuredness of the mesh). Also, from the existing results in $[5,11,12,20]$, one can see that the $\Theta_{2}$ term is strongly dependent on the unstructuredness of the mesh. However, based on [3], the estimate (3.5) suggests that there is a trend that $\Theta_{2}$ decreases with the scaling $H$. See Fig. 2 for numerical evidence.

In this paper, we seek to obtain the minimized error of the filtered solution with respect to the scaling order $\mu$. To do this, we need to find a proper scaling order $\mu$ (assuming $H=h^{\mu}$ ) such that $\Theta_{1}=\Theta_{2}$. As mentioned in [3], in the worst case the scaling order $\mu=\mu_{0}=\frac{2 k+1}{3 k+2} \geq 0.6$, and in the best case $\mu \approx 1$. We examine the $L^{2}$ and $L^{\infty}$ errors with scaling order $\mu$ in the range of $[0.6,1]$ for two nonuniform meshes: Meshs 2.1 and 2.2. Figure 2 shows the variations when $\mu$ increases from 0.6: the error is first reduced until a minimum error is achieved and then the error starts to rise again. We can see that the minimized error in the $L^{2}$ and $L^{\infty}$ norms correspond to the different scaling orders $\mu$; see also Table 2. Since the theoretical estimates are based on the $L^{2}$ norm, in the following we define the concept of the optimal accuracy based on the $L^{2}$ norm.

Definition 3.1 (Optimal Accuracy) For a given mesh, the optimal accuracy of the filtered solution is given by

$$
\min _{0 \leq H \leq 1}\left\|u-K_{H}^{(2 k+1, k+1)} \star u_{h}\right\|_{0} .
$$

The scaling $H$ that minimizes the error is referred to as the optimal scaling and denoted as $H^{\star}$, where the optimal scaling order $\mu^{\star}$ is defined as $H^{\star}=h^{\mu^{\star}}$. Note:

- When $H=0$, the filter $K_{H}^{(2 k+1, k+1)}$ degenerates to the delta function and we have

$$
\left\|u-K_{H}^{(2 k+1, k+1)} \star u_{h}\right\|_{0}=\left\|u-\delta \star u_{h}\right\|_{0}=\left\|u-u_{h}\right\|_{0} .
$$

In this sense, the optimal accuracy is at least as good as the original DG accuracy.

- Since $H \in[0,1]$ and $\left\|u-K_{H}^{(2 k+1, k+1)} \star u_{h}\right\|_{0}$ is continuous respect to $H$, the minimum of (3.6) must exist.

Remark 3.1 We can also define the optimal accuracy based on different norms, such as the $L^{\infty}$-norm, or even different filters, but it will lead to different optimal scaling order $\mu^{\star}$. 

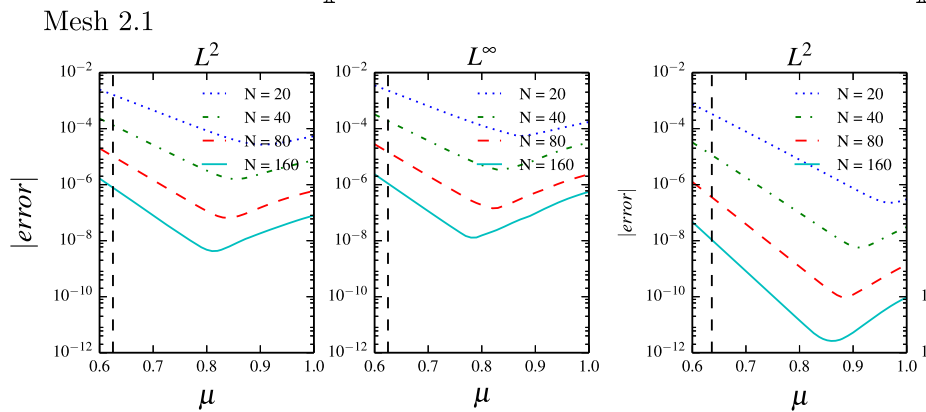

$\mathbb{P}^{3}$

$\mathbb{P}^{2}$

Mesh 2.2
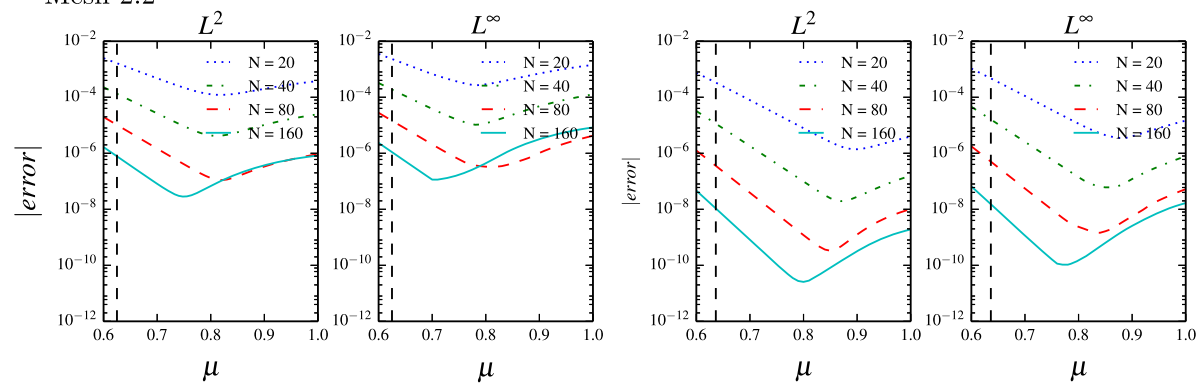

Fig. 2 The $L^{2}$ and $L^{\infty}$ errors in $\log$ scale of the filtered solutions with various scaling $H=h^{\mu}, \mu \in[0.6,1.0]$. The black dashed line marks the location of $\mu_{0}=\frac{2 k+1}{3 k+2}$. The DG approximation is for the linear equation (5.1) with polynomials of degree $k=2,3$ for Meshs 2.1 and 2.2

Table 2 The optimal scaling order $\mu^{\star}$ with respect to Meshs 2.1 and 2.2 with $N=20,40,80,160$, based on the linear equation (5.1) with periodic boundary conditions

\begin{tabular}{|c|c|c|c|c|c|c|c|c|c|c|}
\hline \multirow{3}{*}{$\begin{array}{l}\text { Mesh } \\
\mathrm{N}\end{array}$} & \multicolumn{5}{|l|}{ Mesh 2.1} & \multicolumn{5}{|l|}{ Mesh 2.2} \\
\hline & \multicolumn{2}{|l|}{$u_{h}$} & \multicolumn{3}{|l|}{$\underline{\boldsymbol{u}_{h}^{\star}}$} & \multicolumn{2}{|l|}{$u_{h}$} & \multicolumn{3}{|l|}{$\underline{\boldsymbol{u}_{h}^{\star}}$} \\
\hline & $L^{2}$ error & Order & $\mu^{\star}$ & $L^{2}$ error & Order & $L^{2}$ error & Order & $\mu^{\star}$ & $L^{2}$ error & Order \\
\hline \multicolumn{11}{|l|}{$\mathbb{P}^{2}$} \\
\hline 20 & $2.62 \mathrm{E}-04$ & - & 0.90 & $2.69 \mathrm{E}-05$ & - & $8.01 \mathrm{E}-04$ & - & 0.82 & $1.21 \mathrm{E}-04$ & - \\
\hline 40 & $3.26 \mathrm{E}-05$ & 3.00 & 0.85 & $1.58 \mathrm{E}-06$ & 4.08 & $6.30 \mathrm{E}-05$ & 3.67 & 0.81 & $4.16 \mathrm{E}-06$ & 4.87 \\
\hline 80 & $3.23 \mathrm{E}-06$ & 3.34 & 0.84 & $6.50 \mathrm{E}-08$ & 4.61 & $3.86 \mathrm{E}-06$ & 4.03 & 0.82 & $1.10 \mathrm{E}-07$ & 5.24 \\
\hline 160 & $4.03 \mathrm{E}-07$ & 3.00 & 0.81 & $4.25 \mathrm{E}-09$ & 3.94 & $1.43 \mathrm{E}-06$ & 1.44 & 0.75 & $2.84 \mathrm{E}-08$ & 1.96 \\
\hline \multicolumn{11}{|l|}{$\mathbb{P}^{3}$} \\
\hline 20 & $7.31 \mathrm{E}-06$ & - & 0.97 & $2.25 \mathrm{E}-07$ & - & $2.07 \mathrm{E}-05$ & - & 0.90 & $1.39 \mathrm{E}-06$ & - \\
\hline 40 & $5.23 \mathrm{E}-07$ & 3.80 & 0.91 & $5.69 \mathrm{E}-09$ & 5.31 & $9.49 \mathrm{E}-07$ & 4.45 & 0.87 & $1.95 \mathrm{E}-08$ & 6.16 \\
\hline 80 & $2.64 \mathrm{E}-08$ & 4.31 & 0.88 & $9.46 \mathrm{E}-11$ & 5.91 & 7.12E-08 & 3.74 & 0.85 & $3.31 \mathrm{E}-10$ & 5.88 \\
\hline 160 & $1.58 \mathrm{E}-09$ & 4.07 & 0.86 & $2.65 \mathrm{E}-12$ & 5.16 & $5.77 \mathrm{E}-09$ & 3.63 & 0.80 & $2.56 \mathrm{E}-11$ & 3.69 \\
\hline
\end{tabular}




\subsubsection{The Convergence Rate}

In Fig. 2, plots of the $L^{2}$ and $L^{\infty}$ error versus the scaling $h^{\mu}$ are given for $0.6<\mu \leq 1$. A dashed line is given at the value $\mu_{0}=\frac{2 k+1}{3 k+2}$. We remind the reader that based on (3.3), the design of the filter leads to

$$
\Theta_{1} \sim \mathcal{O}\left(H^{2 k+2}\right) .
$$

When $\mu$ is decreasing, $H=h^{\mu}$ is increasing, then the $\Theta_{1}$ term becomes dominant once $\mu$ becomes small. We can also observe this from Fig. 2, once $\mu<\mu^{\star}$, the errors of the filtered solutions are dominated by the $\Theta_{1}$ term in (3.3), which has a convergence rate of $\mu(2 k+2)$ (before the minimum occurs in the convergence plots). Table 7 show the results of using $\mu$ such that $\mu_{0}<\mu<\mu^{\star}$. However, as we mentioned earlier, the $\Theta_{2}$ term (Eq. (3.4)) is challenging. Figure 2 demonstrates once $\mu>\mu^{\star}$, the errors of the filtered solutions have a trend to increase with $\mu$, which means the $\Theta_{2}$ has the same trend to increase for $\mu^{\star}<\mu<1$ (if $\mu \rightarrow \infty$, the filtered errors degenerate to the DG errors). In short, Fig. 2 together with Tables 2 and 7 show that with a proper scaling (or scaling order $\mu$ ), the filtered solutions have a higher accuracy order, and the errors are reduced compared to the original DG solutions. We also compare the results to the filtered solutions that use a scaling order $\mu_{0}$ to demonstrate the improvement of using scaling order $\mu>\mu_{0}$. Further, we point out that for the different nonuniform meshes, the value of $\mu^{\star}$ will be different, see Fig. 2 . In the next section, we will mainly concentrate on the given nonuniform mesh only, to find the optimal accuracy (or $\mu^{\star}$ ) of the filtered solutions over the given nonuniform mesh.

\section{The Unstructuredness of Nonuniform Meshes}

In Sect. 3, we proposed the concept of the optimal accuracy and numerically demonstrated that there exists an optimal scaling order $\mu^{\star}$ such that using the optimal scaling, $H^{\star}=h^{\mu^{\star}}$, minimizes the error of the filtered solutions in the $L^{2}$ norm. Then, the remaining question is how to find $\mu^{\star}$ for a given nonuniform mesh. Table 2 provides $\mu^{\star}$ by testing different values of the scaling, which is certainly impractical. Theoretically, even for uniform meshes whose optimal scaling order is $\mu^{\star} \approx 1$, it is impossible to find the exact value of $\mu^{\star}$. However, in this section, we propose an approximation $\mu_{h}$ that is sufficiently close to $\mu^{\star}$ and leads to filtered solutions with improved quality.

An important observation from Fig. 2 for determining $\mu^{\star}$ is that the optimal scaling order depends on the structure of the nonuniform meshes, and hence the optimal scaling order is different. The rule of thumb is that the more unstructured the mesh, the smaller the value of $\mu^{\star}$. In order to approximate the value of $\mu^{\star}$, it is important to define a measure of the unstructuredness of nonuniform meshes.

\subsection{The Measure of Unstructuredness of Nonuniform Meshes}

Before discussing the unstructuredness, we first provide a definition of structured meshes.

Definition 4.1 (Structured Mesh) A mesh with $N$ elements is considered structured if there exists a function $f \in \mathcal{C}^{\infty}$ and $f^{\prime}>0$, such that

$$
x_{j+\frac{1}{2}}=f\left(\xi_{j+\frac{1}{2}}\right), \quad \forall j=0, \ldots, N,
$$


where $\left\{\xi_{j+\frac{1}{2}}\right\}_{j=0}^{N}$ corresponds to a uniform mesh with $N$ elements over the same domain.

According to [20], filtered solutions for structured meshes have the same accuracy order $(2 k+1$ for linear hyperbolic equations) as for uniform meshes.

Now we introduce a new parameter $\sigma$, the unstructuredness of the nonuniform mesh, to measure the difference between the given nonuniform mesh and a structured mesh with the same number of elements.

Definition 4.2 (Unstructuredness) For a nonuniform mesh $\left\{x_{j+\frac{1}{2}}\right\}_{j=0}^{N}$, its unstructuredness $\sigma$ is given by

$$
\sigma=\inf _{f \in \mathcal{C}^{\infty}, f^{\prime}>0}\left(\sum_{j=0}^{N}\left(f\left(\xi_{j+\frac{1}{2}}\right)-x_{j+\frac{1}{2}}\right)^{2} /(N+1)\right)^{\frac{1}{2}}
$$

where $\left\{\xi_{j+\frac{1}{2}}\right\}_{j=0}^{N}$ corresponds to the uniform mesh with $N$ elements for the same domain. The smaller the $\sigma$, the more structured the mesh. have

Without loss of generality, we denote the domain $\Omega=[0,1]$. Then, in the worst case, we

$$
\left(\sum_{j=0}^{N} \frac{\left(f\left(\xi_{j+\frac{1}{2}}\right)-x_{j+\frac{1}{2}}\right)^{2}}{N+1}\right)^{\frac{1}{2}}<\left(\sum_{j=0}^{N} \frac{(1-0)^{2}}{N+1}\right)^{\frac{1}{2}}=1 \Rightarrow \sigma<1 .
$$

Remark 4.1 The definition of unstructuredness is designed by considering the discrete $L^{2}$ norm formula. It is a natural choice since the focus is on the error in the $L^{2}$ norm. Furthermore, it establishes a connection between general nonuniform meshes and the well-studied structured meshes. Besides formula (4.2), there are different ways to identify the unstructuredness of the mesh, such as through the variation of mesh elements [8], utilizing different norms, or the methods mentioned in Appendix.

\subsection{SIAC Filtering Based on Unstructuredness}

After defining the unstructuredness, $\sigma$, we now study the relation of $\sigma$ and the filter scaling, which allows for determining $\mu_{h}$. This depends on two very challenging estimates: that of the negative-order norm and that of the divided differences over a nonuniform mesh. Note that for the divided difference with a general scaling $H, u_{h}\left(x+\frac{H}{2}\right)$ and $u_{h}\left(x-\frac{H}{2}\right)$ are not in the same approximation space even for uniform meshes. Since the translation invariance with respect to both the DG mesh size $h$ and the scaling $H$, for uniform meshes, one has to let the scaling $H$ satisfies that $H=m h$ ( $m$ is a positive integer) to keep $u_{h}\left(x+\frac{H}{2}\right)$ and $u_{h}\left(x-\frac{H}{2}\right)$ in the same space. Therefore, it is difficult to establish a rigorous error estimates. In Theorem 3.1, a rough error estimate of $\partial_{H} u_{h}$ is obtained by using the bound

$$
\begin{aligned}
\left\|\partial_{H}\left(u-u_{h}\right)\right\|_{0} & \leq \frac{1}{H}\left(\left\|\left(u-u_{h}\right)\left(x+\frac{H}{2}\right)\right\|_{0}+\left\|\left(u-u_{h}\right)\left(x-\frac{H}{2}\right)\right\|_{0}\right) \\
& \leq \frac{2}{H}\left\|u-u_{h}\right\|_{0} .
\end{aligned}
$$


This does not take into the unique unstructuredness of a given mesh. Further, as demonstrated in the previous section, the result is not optimal. Here, in this paper, we are seeking a robust algorithm which is useful in a practical setting to obtain error reduction.

In this section, we propose a method based on relating the nonuniform mesh to its closest structured mesh (under Definition (4.2)). That is,

$$
\underbrace{\left\|\partial_{H}\left(u-u_{h}\right)\right\|_{0}}_{\text {nonuniform mesh }} \leq \underbrace{\left\|\partial_{H}\left(u-u_{h}\right)\right\|_{0, f(\xi)}}_{\text {structured mesh }}+\underbrace{\left\|\partial_{H}\left(u-u_{h}\right)\right\|_{0, \text { diff }}}_{\text {difference }} .
$$

As mentioned earlier [20], we know that the first divided difference over the structured mesh $\left\{f\left(\xi_{j+\frac{1}{2}}\right)\right\}_{j=0}^{N}$ has nice properties. Then, we assume that the error of the first divided difference of the DG solution for the nonuniform mesh $\left\{x_{j+\frac{1}{2}}\right\}_{j=0}^{N}$ is dominated by the difference between the nonuniform mesh and its closest structured mesh.

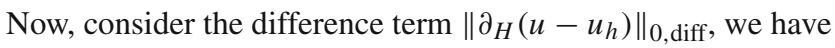

$$
\left\|\partial_{H}\left(u-u_{h}\right)\right\|_{0, \operatorname{diff}}=\frac{2}{H}\left(\sum_{j=0}^{N}\left\|u-u_{h}\right\|_{0, \Omega_{j}}^{2} /(N+1)\right)^{\frac{1}{2}},
$$

where $\Omega_{j}=\left[x_{j+\frac{1}{2}}, f\left(\xi_{j+\frac{1}{2}}\right)\right]$ (or $\Omega_{j}=\left[f\left(\xi_{j+\frac{1}{2}}\right), x_{j+\frac{1}{2}}\right]$ ). Since the approximation $u_{h}$ on the interval $\Omega_{j}$ cannot be estimated rigorously through the traditional error estimates, we assume that

$$
\begin{aligned}
\left\|u-u_{h}\right\|_{0, \Omega_{j}}^{2}=\int_{\Omega_{j}}\left(u-u_{h}\right)^{2} d x & \leq C\left|\Omega_{j}\right|\left\|u-u_{h}\right\|_{\infty}^{2} \\
& =C\left|x_{j+\frac{1}{2}}-f\left(\xi_{j+\frac{1}{2}}\right)\right| h^{2 k+2} .
\end{aligned}
$$

The above assumption is based on $L^{\infty}$ estimate that

$$
\left\|u-u_{h}\right\|_{\infty} \leq C h^{k+1},
$$

which has not been proven theoretically, but validate numerically for rectangular meshes (the meshes considered in this paper). For general unstructured triangular meshes, a reduced accuracy order of $\mathcal{O}\left(h^{k+1-\frac{d}{2}}\right)$ needs to be considered. Then, by using the Cauchy-Schwarz inequality, we have

$$
\begin{aligned}
\left\|\partial_{H}\left(u-u_{h}\right)\right\|_{0, \text { diff }} & =\frac{2}{H}\left(\sum_{j=0}^{N}\left\|u-u_{h}\right\|_{0, \Omega_{j}}^{2} /(N+1)\right)^{\frac{1}{2}} \\
& \leq C h^{k+1} H^{-1}\left(\sum_{j=0}^{N}\left|x_{j+\frac{1}{2}}-f\left(\xi_{j+\frac{1}{2}}\right)\right| /(N+1)\right)^{\frac{1}{2}} \\
& =C h^{k+1} H^{-1}\left\{\left(\sum_{j=0}^{N}\left(f\left(\xi_{j+\frac{1}{2}}\right)-x_{j+\frac{1}{2}}\right)^{2} /(N+1)\right)^{\frac{1}{2}}\right\}^{\frac{1}{2}} .
\end{aligned}
$$


By using Definition (4.2) and the assumption that $\left\|\partial_{H}\left(u-u_{h}\right)\right\|_{0 \text {, diff }}$ is the dominant term, we obtain

$$
\left\|\partial_{H}\left(u-u_{h}\right)\right\|_{0} \leq C \frac{\sqrt{\sigma}}{H} h^{k+1}=C \frac{h^{\frac{1}{2} \log _{h} \sigma}}{H} h^{k+1},
$$

and by induction

$$
\left\|\partial_{H}^{\alpha}\left(u-u_{h}\right)\right\|_{0} \leq C \frac{\sqrt{\sigma}}{H} h^{k+1}=C\left(\frac{h^{\frac{1}{2} \log _{h} \sigma}}{H}\right)^{\alpha} h^{k+1} .
$$

Remark 4.2 The above analysis is the motivation for using formula (4.2) to define the unstructuredness. Also, we point out that assumption (4.3) is an empirical rather than a rigorous

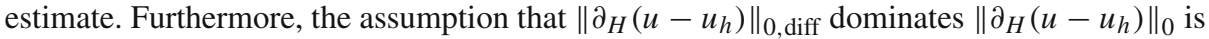
reasonable only when the nonuniform mesh is not so close to the respective structured mesh $(\sigma \gg 0)$.

Based on the value of $\sigma$, we divide the nonuniform meshes into two groups and discuss the corresponding strategies separately.

- Nearly structured meshes $\log _{h} \sigma \geq 2$.

This definition is based on estimate (4.5), when

$$
\frac{\sqrt{\sigma}}{h} \geq \frac{\sqrt{\sigma}}{H} \geq 1, \quad \Rightarrow \sigma \geq h^{2} \Rightarrow \log _{h} \sigma \geq 2 .
$$

Then, the nonuniform mesh is almost a structured mesh, and the effect of the difference is negligible. In other words, we can treat these nearly structured meshes as structured meshes and use the conclusions in [20]. Also, we note that the definition is not strict; when $\log _{h} \sigma \approx 2$ we can also treat these nonuniform meshes as structured meshes.

- Unstructured meshes $\log _{h} \sigma<2$.

This is a more challenging case and the aim of this paper. Under the same conditions as in Lemma 2.2, we assume that for a nonuniform mesh with the unstructuredness parameter $\sigma$ as defined in Eq. (4.2) and based on the results in [22], the divided differences of DG solution satisfies

$$
\left\|\partial_{H}^{\alpha}\left(u-u_{h}\right)\right\|_{-(k+1), \Omega_{0}} \leq C h^{2 k+1}\left(\frac{h^{\frac{1}{2} \log _{h} \sigma}}{H}\right)^{\alpha},
$$

when $H \leq h^{\frac{1}{2} \log _{h} \sigma}$. Moreover, the divided differences of the approximation satisfy

$$
\sum_{\alpha=0}^{k+1}\left\|\partial_{H}^{\alpha}\left(u-u_{h}\right)\right\|_{-(k+1)} \leq C\left(\frac{h^{\frac{1}{2} \log _{h} \sigma}}{H}\right)^{k+1} h^{2 k+1},
$$

and according using the estimates for the filter design and and approximation (Eqs. (3.2)(3.4)), we can enforce

$$
H^{2 k+2}=\left(\frac{h^{\frac{1}{2} \log _{h} \sigma}}{H}\right)^{k+1} h^{2 k+1} .
$$

Using $H=h^{\mu_{h}}$, we then have for $\mu_{h}$ that

$$
\mu_{h}=\frac{2 k+1}{3(k+1)}+\frac{1}{6} \log _{h} \sigma \approx \frac{2}{3}+\frac{1}{6} \log _{h} \sigma>\frac{1}{2} \log _{h} \sigma,
$$


Table 3 Four types of scaling order used in the performance validation

\begin{tabular}{|c|c|}
\hline Scaling order & Definition \\
\hline$\mu_{0}$ & $\mu_{0}=\frac{2 k+1}{3 k+2}$, see Theorem 3.1 \\
\hline$\mu_{\max }$ & $h^{\mu_{\max }}=\max \Delta x_{j}, j=1, \ldots, N$ \\
\hline$\mu_{h}$ & $\mu_{h}=\frac{2 k+1}{3(k+1)}+\frac{1}{6} \log _{h} \sigma \approx \frac{2}{3}+\frac{1}{6} \log _{h} \sigma$, see (4.7) \\
\hline$\mu^{\star}$ & $H=h^{\mu^{\star}}$ minimizes $\left\|u-K_{H}^{(2 k+1, k+1)} \star u_{h}\right\|_{0}$ \\
\hline
\end{tabular}

which is much more reasonable to compute as $H=h^{\mu_{h}} \leq h^{\frac{1}{2} \log _{h} \sigma}$.

\subsection{Scaling Performance Validation}

At the beginning of this section, we first summarize the definitions of all the scalings that are going to be tested in the section, see Table 3.

As mentioned in Sect. 3, Theorem 3.1 is not practical since the

- the accuracy order improvement requires $k \geq 2$;

- the errors in the DG solution are not always reduced.

In order to construct a robust algorithm that can be used in practice, we have proposed using scaling (4.7), which demonstrates the relation of the scaling order $\mu_{h}$ and the unstructuredness, $\sigma$. Since this result is not based on a rigorous error estimate, in this section, we validate the performance of the proposed scaling $H=h^{\mu_{h}}$, where $\mu_{h}$ is given in Eq. (4.7) by testing it for many nonuniform meshes. For a fair demonstration, we also compared this scaling with the scaling provided by Theorem 3.1 and the maximum scaling used in many works, such as $[5,12]$. For convenience, we use the corresponding scaling orders $\mu_{h}, \mu_{0}$ and $\mu_{\max }$ to refer these three strategies, respectively (see Table 3).

\subsubsection{Test Set-up}

First, we present the setting of the nonuniform meshes used for the performance test. Since nearly structured meshes are relatively easily studied, in this test, we focus on unstructured meshes (or meshes with random structures). The information is presented as follows:

- We adopt Mesh 2.2 with $b=0.3$. The value of $b$ is chosen not only for allowing sufficient generality of the mesh structure, but also in order to avoid the possibility of round-off error caused by tiny elements.

- In this test, we have considered the number of elements $N=20,40,80$, using 1700 different samples (5100 meshes in total).

- The finer meshes $(N=40,80)$ are generated using rules similar to the coarse mesh ( $N=20$ ), which preserves the nonuniform property. A trivial way to generating the finer mesh is by uniformly refining the coarse mesh, which leads to piecewise uniform meshes when $N$ is large. 

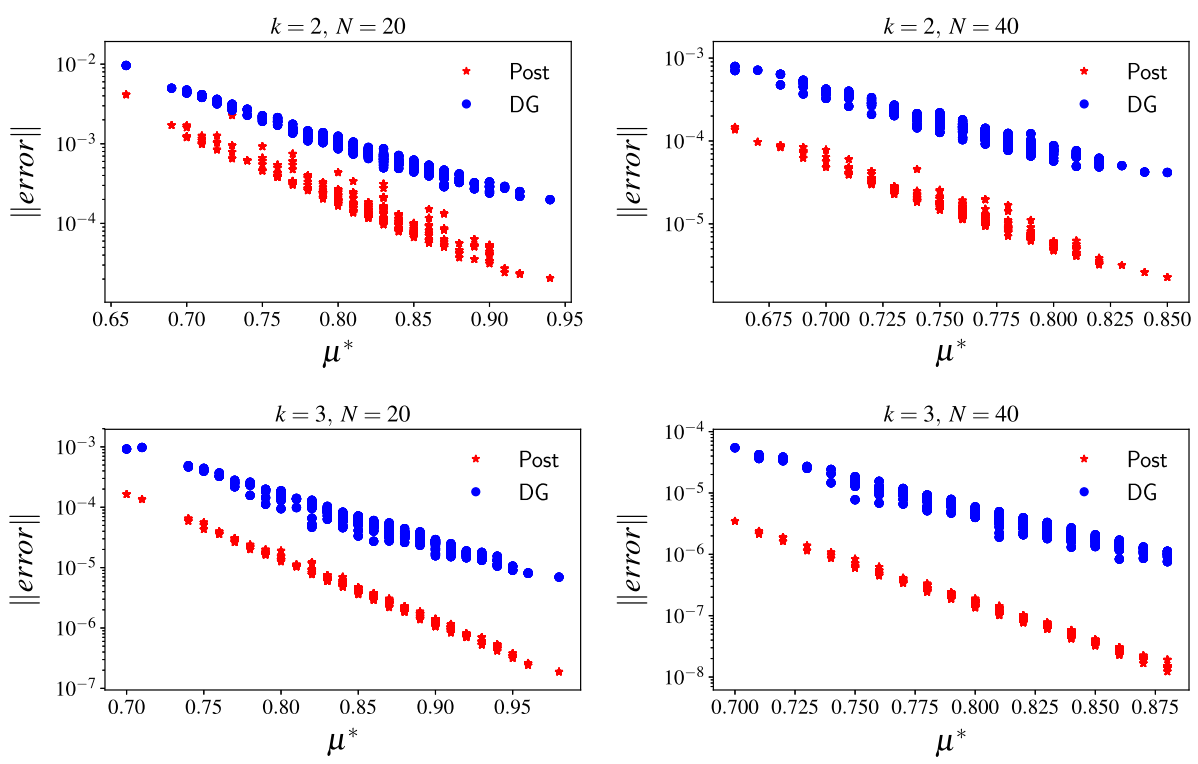

Fig. 3 The comparison of DG errors and their optimal filtered results for different nonuniform meshes respect to $\mu^{\star}$. Each plot is based on 1700 random nonuniform samples

\subsubsection{Optimal Scaling Order $\mu$ Versus Errors}

We begin by examining how the optimal scaling order $\mu^{\star}$ and the filtered solutions are altered with the DG approximation over different nonuniform meshes (shows as different DG solutions). This relation is demonstrated in Fig. 3. Notice the following:

- Trend 1: A larger $\mu^{\star}$, corresponds to a smaller filtering region and lower errors for filtered solution. The lower errors clearly displayed for $k=3$ than $k=2$. It also corresponds to a more structured mesh as well.

- Trend 2: Also demonstrated is that when the errors are lower for the DG solution, the optimal filtered solution has better error. This fact is supported by the theory.

- Trend 3: Notice that $\mu_{0}=\frac{2 k+1}{3 k+2}$ is approximately 0.63 and 0.64 for $k=2,3$. However, we can see that in most cases, this value is far away from $\mu^{\star}$.

\subsubsection{Optimal Scaling versus Existing Scalings}

After checking our test meshes for the optimal scaling, we check the performance of the existing scalings and compare the results with the optimal filtered solution. In Fig. 4, the ratio of the $L^{2}$-errors for the DG solution to the $L^{2}$-errors for the filtered solution are plotted against the probability of achieving that ratio for a given polynomial order and mesh. If the ratio is less than one then the filtered error is better than the DG error, in other words, the filtered solution is at least accuracy-conserving compared to the DG solution. Further, by considering the ratio of the DG error to the SIAC Filtered error (Fig. 4), one can see that the performance (the ratio) of the SIAC filtering varies with the approximation over different nonuniform mesh approximations. On the other hand, we can compare the performance of different scalings by comparing their histogram plots (Fig. 4). One can tell that one scaling 

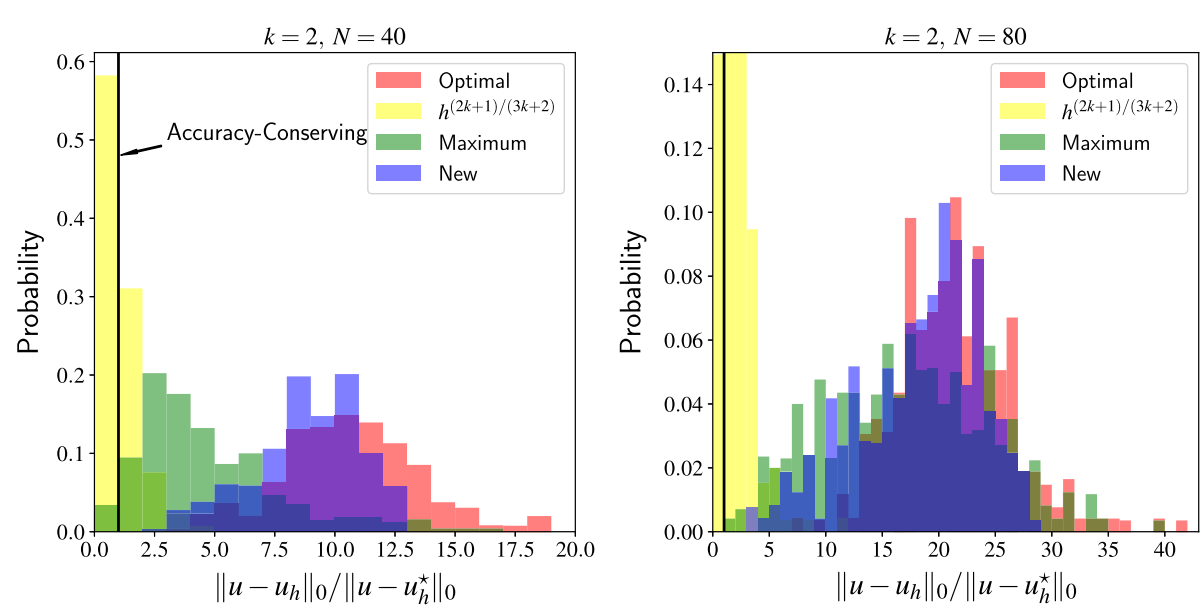

Fig. 4 The comparison for the performance of different scalings: optimal scaling, theoretical scaling, maximum scaling, the new scaling for $k=2$. The $\mathrm{x}$-axis is the value of $\left\|u-u_{h}\right\|_{0} /\left\|u-u_{h}^{\star}\right\|_{0}$, clearly, the larger the value, the better the filtering. In addition, we mark the accuracy-conserving position $x=1$ with a black line

has a histogram closer to the optimal scaling (red) and also has the better performance. Here, we remind the reader that the different scalings are given in Table 3.

- Theoretical Scaling, $\mu_{0}$ (yellow): For more than half of the mesh samples, the ratio between the DG error and filtered error remains relatively small and the probability of achieving this scaling is higher than for other scalings.

- Maximum Scaling, $\mu_{\max }$ (green): This scaling produces a reasonable ratio for most situations.

- proposed Scaling, $\mu_{h}$ (purple): The performance is closer to the optimal results compared to the other two scaling.

Remark 4.3 We note that the value of $\mu^{\star}$ is also affected by the exact solution $u$, more precisely $\frac{|u|_{H^{2 k+2}}}{|u|_{H^{k+1}}}$. Since the exact solution is usually unknown in practice, this is difficult to determine. However, this leads us to choose $\mu_{h}$ to be slight smaller than $\mu^{*}$.

\subsubsection{Comparisons}

From Fig. 4, We can clearly see that the new proposed scaling order $\mu_{h}$ has the best performance. Now, we use the statistical data of results to give a more clear view of the performance.

First, we check the basic accuracy-conserving property in order to ensure that we are not degrading the DG results. From Table 4, we can see that $\mu_{h}$ performs the best with respect to accuracy conservation, $\mu_{0}$ the worst one, and $\mu_{\max }$ still has considerably large problems for coarse meshes.

Next, we compare the proposed scaling with other two scalings side-by-side in Tables 5 and 6 . Here, motivated by the definition of equivalence of norms, we add the category "similar" to account for small differences in results: if error 1 and error 2 satisfy that $\frac{1}{C_{\text {tol }}} \mid$ error $_{1} \mid \leq$ $\mid$ error $_{2}\left|\leq C_{t o l}\right|$ error $_{1} \mid$, then these two errors are counted as similar. In this note, the tolerance constant $C_{t o l}$ is set as 2 .

1. Table $5, \mu_{0}$ versus $\mu_{h}$ : the data clearly suggests that $\mu_{h}$ is a better choice than $\mu_{0}$. 
Table 4 Percent of results which are at least accuracy-conserving $\left(\left\|u-u_{h}^{\star}\right\|_{0} \leq\left\|u-u_{h}\right\|_{0}\right)$

\begin{tabular}{llllll}
\hline Degree & $N$ & $\mu_{0}(\%)$ & $\mu_{\max }(\%)$ & $\mu_{h}(\%)$ & $\mu^{\star}(\%)$ \\
\hline $\mathbb{P}^{2}$ & 20 & 13.5 & 58.9 & 100 & 100 \\
& 40 & 41.8 & 96.6 & 100 & 100 \\
& 80 & 85.1 & 100 & 100 & 100 \\
$\mathbb{P}^{3}$ & 20 & 3.9 & 5.8 & 100 & 100 \\
& 40 & 12.2 & 69.8 & 100 & 100 \\
& 80 & 45.6 & 99.6 & 100 & 100 \\
\hline
\end{tabular}

Table $5 \mu_{0}$ versus $\mu_{h}$

\begin{tabular}{lllll}
\hline \multirow{2}{*}{ Degree } & $N$ & $\mu_{0}$ & & $\mu_{h}$ \\
\cline { 3 - 5 } & $N$ & Better $(\%)$ & Similar $(\%)$ & Better $(\%)$ \\
\hline$p=2$ & 20 & 0.0 & 6.1 & 93.9 \\
& 40 & 0.0 & 4.7 & 95.2 \\
& 80 & 0.8 & 3.9 & 95.3 \\
& 20 & 0.0 & 0.8 & 99.2 \\
& 40 & 0.0 & 0.7 & 99.3 \\
& 80 & 0.0 & 1.2 & 98.8 \\
\hline
\end{tabular}

\begin{tabular}{|c|c|c|c|c|}
\hline \multirow[b]{2}{*}{ Degree } & \multirow[b]{2}{*}{$N$} & \multicolumn{2}{|l|}{$\mu_{\max }$} & \multirow{2}{*}{$\frac{\mu_{h}}{\text { Better }(\%)}$} \\
\hline & & Better (\%) & Similar (\%) & \\
\hline \multirow[t]{3}{*}{$p=2$} & 20 & 0.4 & 16.7 & 82.9 \\
\hline & 40 & 1.2 & 34.9 & 63.9 \\
\hline & 80 & 0.4 & 94.5 & 5.1 \\
\hline \multirow[t]{3}{*}{$p=3$} & 20 & 0.0 & 2.2 & 97.8 \\
\hline & 40 & 0.0 & 7.5 & 92.5 \\
\hline & 80 & 0.4 & 17.9 & 81.7 \\
\hline
\end{tabular}

Table $6 \mu_{\max }$ versus $\mu_{h}$

2. Table $6, \mu_{\max }$ versus $\mu_{h}$ : in at least $98 \%$ of the cases sampled, $\mu_{h}$ produced better results than using $\mu_{\max }$.

Based on the number of samples and the statistical data, the new scaling is a reliably better scaling to use among the scalings discussed in this article.

Through many performance tests, it is reasonable to claim that by using the proposed scaling $\mu_{h}$, we can expect that there is an accuracy improvement for $k \geq 1$ for the given nonuniform mesh (dependent on $\sigma$ ). In practice, strategy 4.7 provides a way to find the proper scaling for the SIAC filter, it can be used to reduce the errors of given DG solutions.

\subsection{A Note on Computation}

Aside from error reduction, the computational cost of using the filter is also an important factor in practical applications. As mentioned in previous sections, the scaling $H$ used in Theorem 3.1 is usually larger than the scaling required for nonuniform meshes, which means that the computational cost is higher than the uniform mesh case [3,11]. Based on Fig. 2, 
when $\mu \in\left[\mu^{\star}, 1\right]$, the final accuracy is directly related to the scaling order $\mu$, which means one can sacrifice accuracy to improve computational efficiency. For example, if the mesh is closer to a structured mesh, a naive choice of scaling $H=\max _{j} \Delta x_{j}$ (or $H=1.5 \max _{j} \Delta x_{j}$, $\left.H=2 \max _{j} \Delta x_{j}\right)$ can lead to acceptable results as obtained in $[5,12]$.

\section{Numerical Results}

In the previous section, we proposed using the scaling order $\mu_{h}$ given by Eq. (4.7). Using the scaling order $\mu_{h}$ can improve the accuracy order and reduce the error from the original discontinuous Galerkin approximation. Also, since $\mu_{h}$ is designed to approximate the optimal scaling order $\mu^{\star}$, the filtered solutions are expected to have a reduction in error compared to the DG approximation. For numerical verification, we apply the newly designed scaling order $\mu_{h}$ for various differential equations over nonuniform meshes-Meshs 2.1 and 2.2and compare it with using scaling order $\mu_{0}$ mentioned in Theorem 3.1. Also, we note that the initial approximation $u_{h}(x, 0)$ is the $L^{2}$ projection of the initial function $u(x, 0)$. The third order TVD Runge-Kutta scheme [7] is used for the time discretization.

\subsection{Linear Equation}

Consider a linear equation

$$
\begin{aligned}
u_{t}+u_{x} & =0, \quad(x, t) \in[0,1] \times(0, T], \\
u(x, 0) & =\sin (2 \pi x),
\end{aligned}
$$

with periodic boundary conditions at time $T=1$ for Meshs 2.1 and 2.2. Table 7 includes the $L^{2}$ and $L^{\infty}$ norm errors of the DG solutions and two filtered solutions with scaling orders $\mu_{0}$ and $\mu_{h}$. First we check the results of using scaling order $\mu_{0}$ in Theorem 3.1. Although the filtered solutions have a better accuracy order, both the $L^{2}$ and $L^{\infty}$ errors are worse than the original DG solution! Theorem 3.1 says something only about the order, but not about the quality of the errors. Using a scaling order $\mu_{h}$, SIAC filtering is able to reduce the errors in the $L^{2}$ and $L^{\infty}$ norm and improve the accuracy order. The filtered errors are reduced compared to the DG errors, especially when using a higher order polynomial or a sufficiently refined mesh. Figure 5, the pointwise error plots, demonstrate the other feature of SIAC filtering as its name implies: smoothness-increasing. Both the filtered solutions are $\mathcal{C}^{k-1}$ functions. The smoothness is significantly improved compared to the weakly continuous DG solutions. To ensure the smoothness of the filtered solution across the entire domain, we consider only a constant scaling $H$ across the entire domain. In Fig. 5 both filtered solutions reduce the oscillations in the DG solution and using a scaling order $\mu_{0}$ completely removes the oscillations due to the large filter support size.

Comparing the results between Meshs 2.1 and 2.2, we can see that the DG solutions and filtered solutions with scaling order $\mu_{h}$ are better for Mesh 2.1 than for Mesh 2.2 because Mesh 2.1 is more structured than Mesh 2.2. However, using scaling order $\mu_{0}$ generates almost the same result, which shows that $\mu_{0}$ does not take the mesh structures into account. 


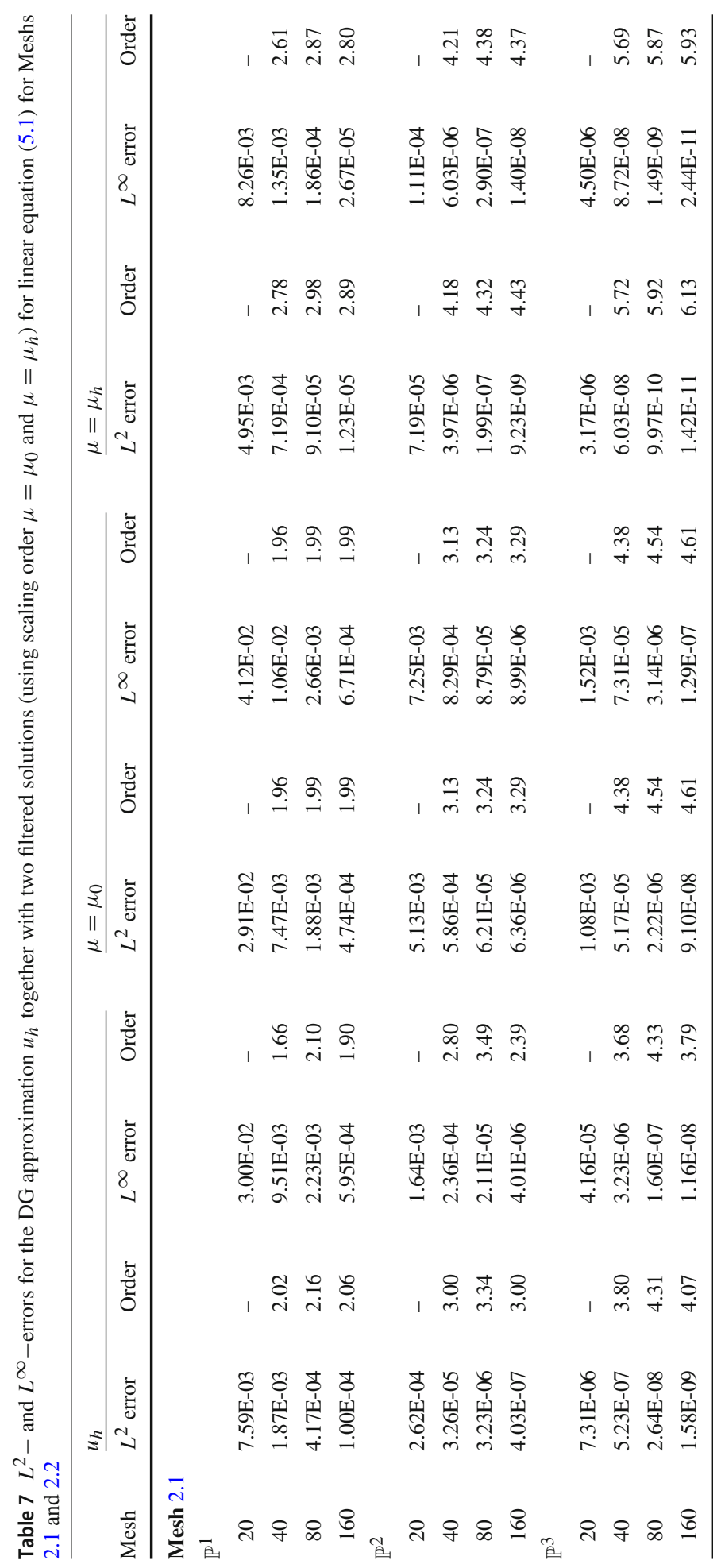




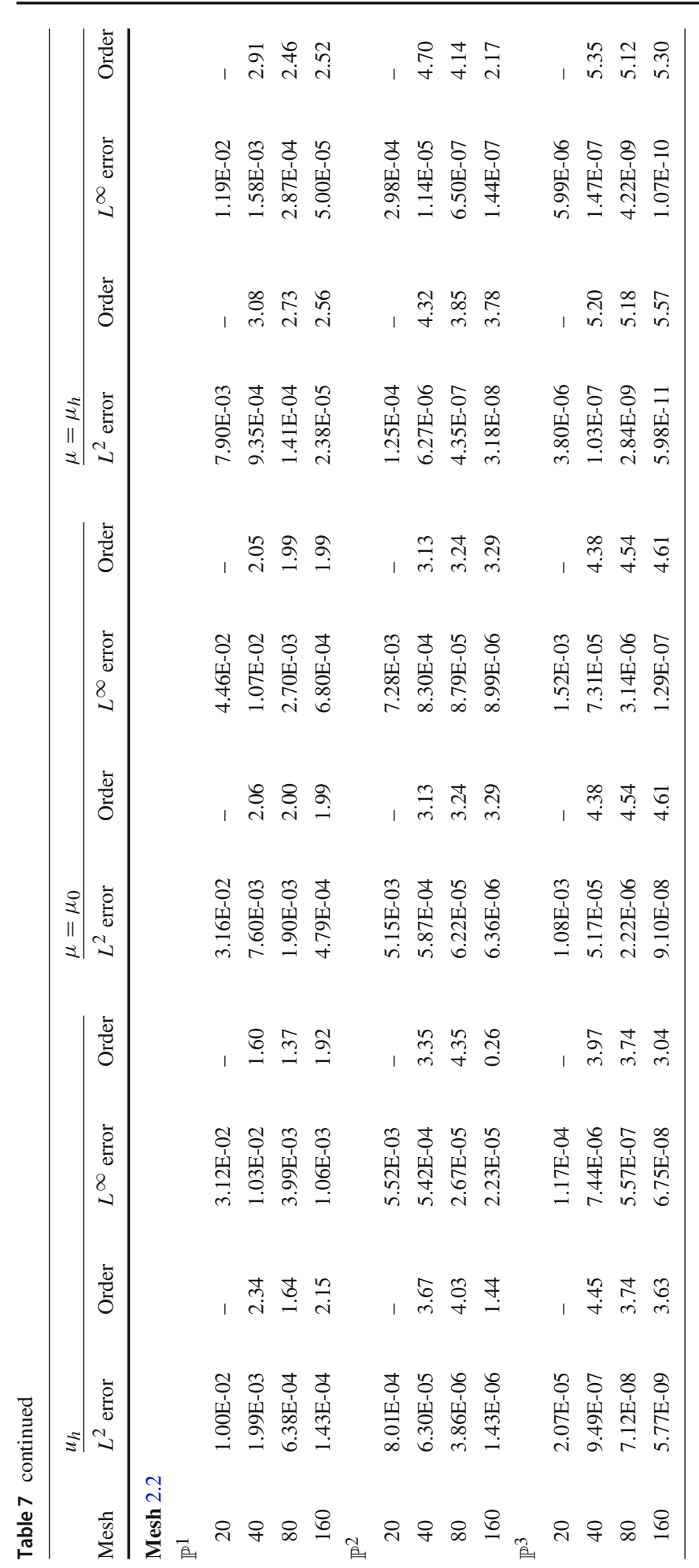


$D G$

Mesh 2.1

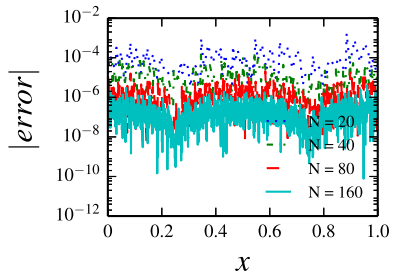

Mesh 2.2

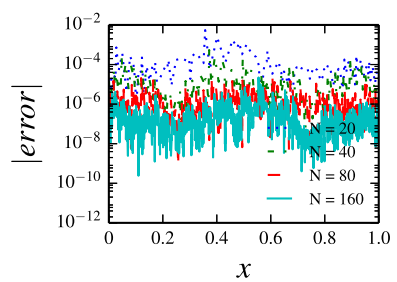

$\mu=\mu_{0}$
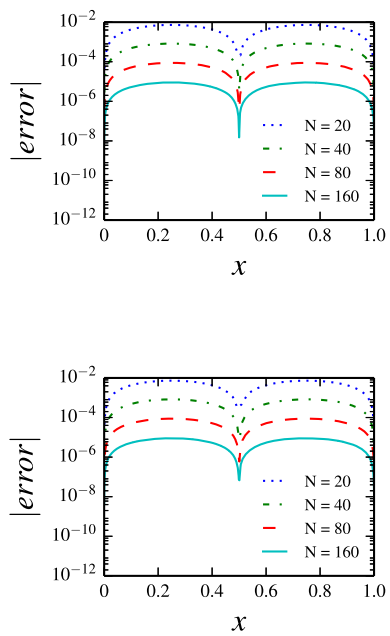

$\mu=\mu_{h}$
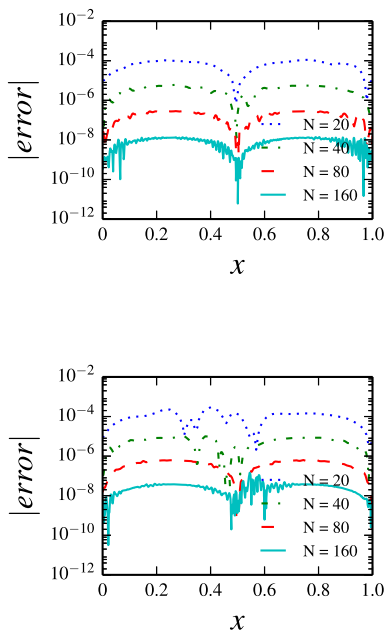

Fig. 5 Comparison of the pointwise errors in log scale of the DG approximation together with two filtered solutions (using scaling order $\mu=\mu_{0}$ and $\mu=\mu_{h}$ ) for linear equation (5.1) for Meshs 2.1 and 2.2 with polynomials of degree $k=2$

\subsection{Variable Coefficient Equation}

After the linear equation (5.1), which has a constant coefficient, we consider the variable coefficient equation

$$
\begin{aligned}
u_{t}+(a u)_{x} & =f, \quad(x, t) \in[0,1] \times(0, T] \\
u(x, 0) & =\sin (2 \pi x),
\end{aligned}
$$

where the variable coefficient $a(x, t)=2+\sin (2 \pi(x+t))$ and the right side term $f(x, t)$ are chosen to make the exact solution be $u(x, t)=\sin (2 \pi(x-t))$. The boundary conditions are periodic and the final time $T=1$.

Similar to the linear equation example, we compare the $L^{2}$ and $L^{\infty}$ norm errors in Table 8. The pointwise error plots are given in Fig. 6 . The results are similar to the previous results for the constant coefficient equation. Here we point out only the features that are different from the linear equation. Using a scaling order $\mu_{0}$ does not reliably reduce the errors in the $L^{2}$ norm and the $L^{\infty}$ norm errors are still worse than the DG solutions. However, using a scaling order $\mu_{h}$ reduces the errors in the $L^{2}$ norm and the $L^{\infty}$ norm. The pointwise error plots in Fig. 6 are more oscillatory compared to Fig. 5 due to the effects of the variable coefficient.

\subsection{Two-Dimensional Example}

For the two-dimensional example, we consider a two-dimensional linear equation

$$
\begin{aligned}
u_{t}+u_{x}+u_{y} & =0, \quad(x, y) \in[0,1] \times[0,1], \\
u(x, y, 0) & =\sin (2 \pi(x+y)),
\end{aligned}
$$




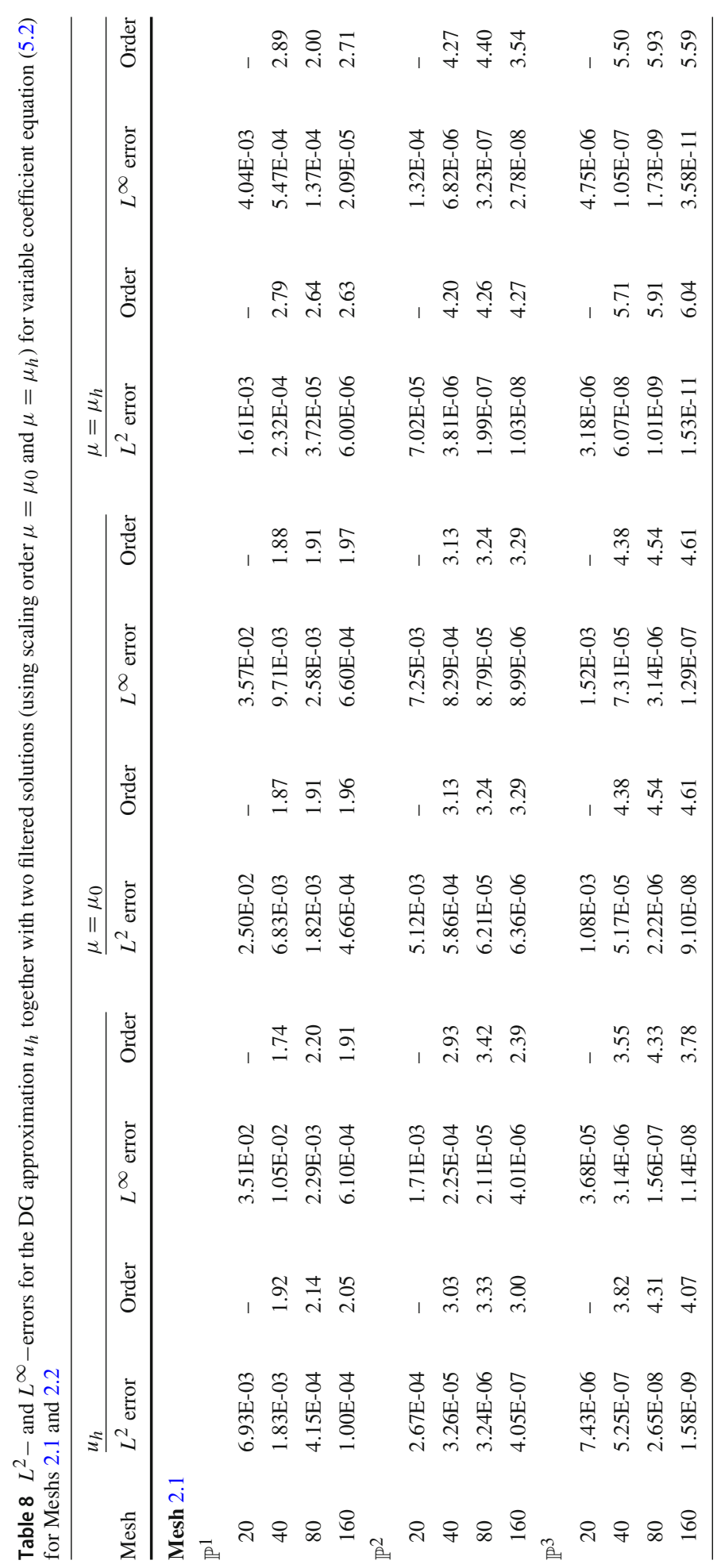




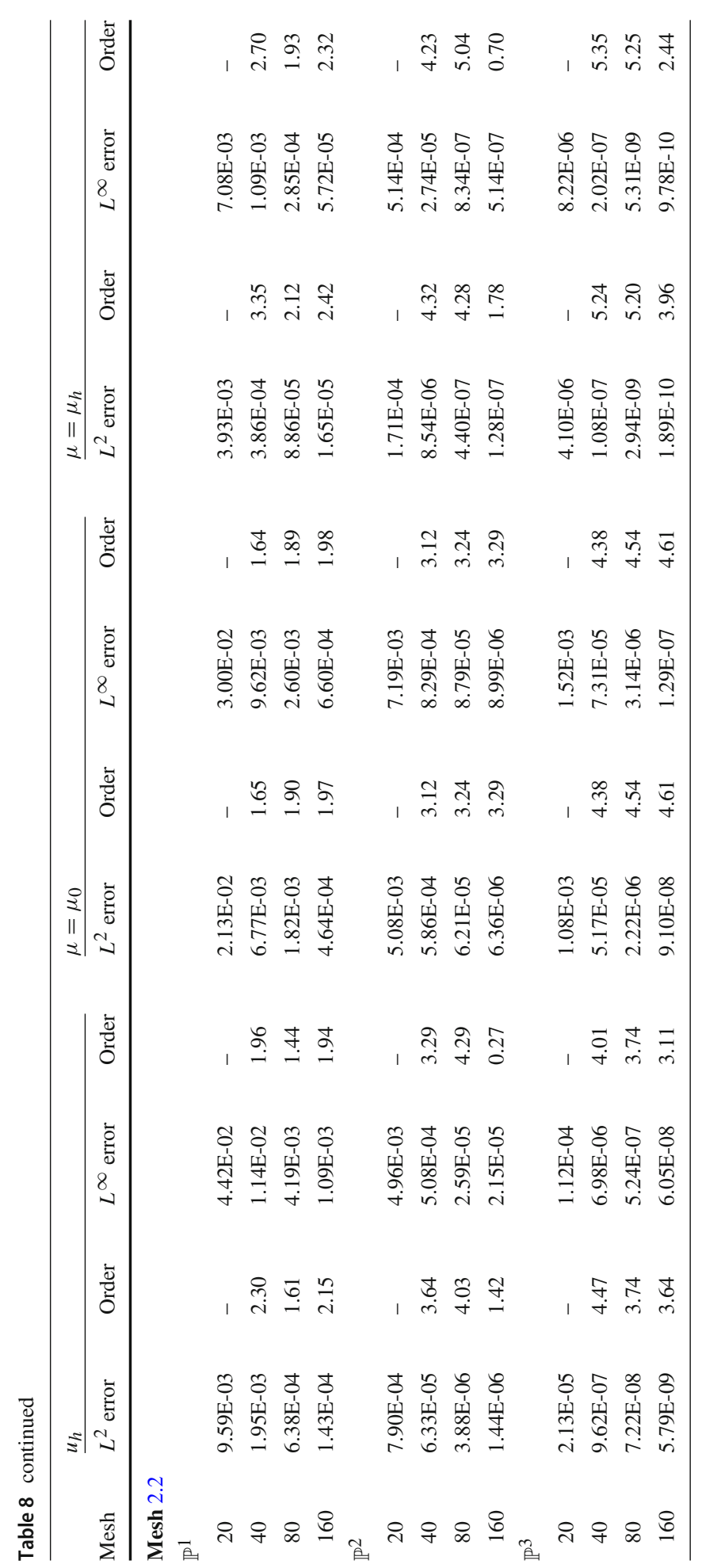


$D G$

Mesh 2.1
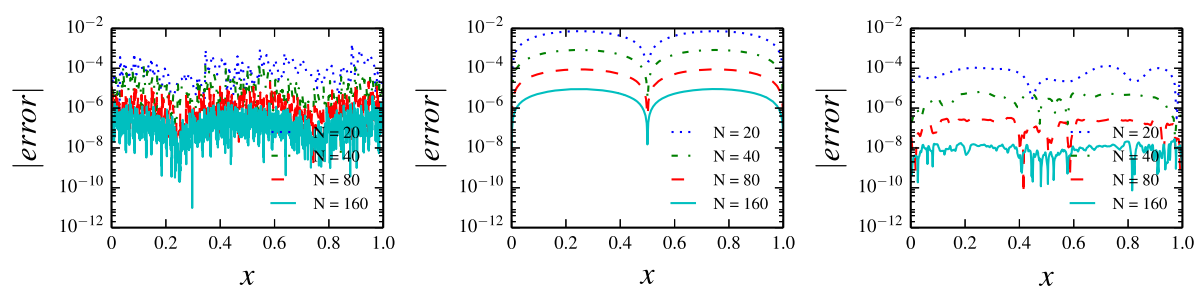

Mesh 2.2
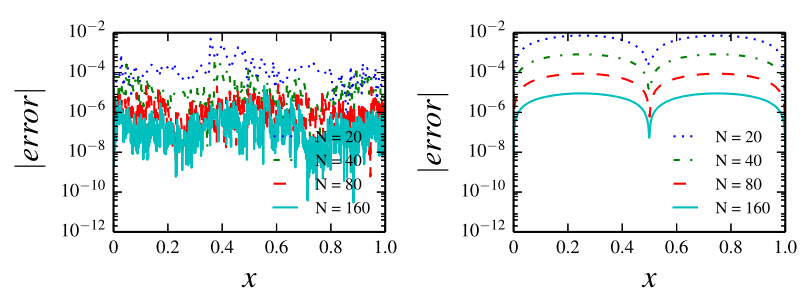

Fig. 6 Comparison of the pointwise errors in log scale of the DG approximation together with two filtered solutions (using scaling order $\mu=\mu_{0}$ and $\mu=\mu_{h}$ ) for variable coefficient equation (5.1) for Meshs 2.1 and 2.2 with polynomials of degree $k=2$

with periodic boundary conditions at time $T=1$ for a two dimensional quadrilateral extension of Meshs 2.1 and 2.2.

The $L^{2}$ and $L^{\infty}$ norm errors are presented in Tables 9 and 10, and the pointwise error plots (pcolor plots) are included in Figs. 7 and 8. The results are very similar to the onedimensional examples: the filtered solutions with scaling order $\mu_{h}$ reduce the errors in the $L^{2}$ norm; using a scaling order $\mu_{0}$ increases the error in the $L^{2}$ norm for the DG error. In the two-dimensional case, computational efficiency becomes more important compared to the one-dimensional case due to the increased computational cost. As mentioned before, using a scaling order $\mu_{0}$ is far more inefficient compared to using the scaling order $\mu_{h}$. In particular, for a $\mathbb{P}^{3}$ polynomial basis with $N=160 \times 160$ meshes, using a scaling order $\mu_{0}$ is more than 8 times slower for Mesh 2.1 (5 times slower for Mesh 2.2) than using the scaling order $\mu_{h}$.

Remark 5.1 In this paper, we only consider periodic boundary conditions. For other boundary conditions such as Dirichlet boundary conditions, a position-dependent filter $[11,20]$ has to be used near the boundaries. The results will be similar to the periodic boundary conditions. However, to obtain the optimal result, a position-dependent scaling has to be applied, we will leave it for the future work.

\section{Conclusion}

In this paper, we have demonstrated that for a given nonuniform mesh, the filtered solution is highly affected by the unstructuredness of the mesh. By adjusting the filter scaling one can minimize the error of the filtered solution. In addition, a scaling $H=h^{\mu_{h}}$ (4.7) of the SIAC filter is proposed in order to approach the optimal accuracy of the filtered solution, where the 


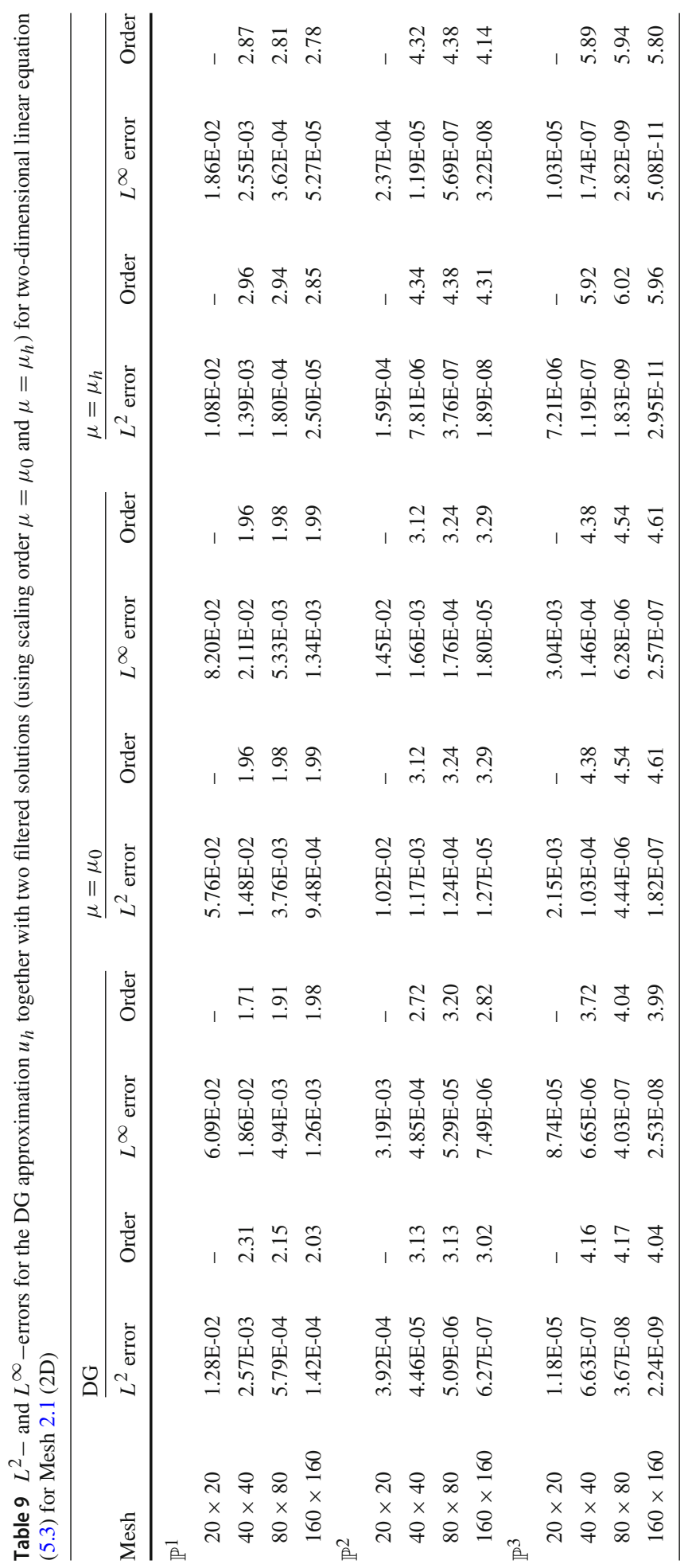




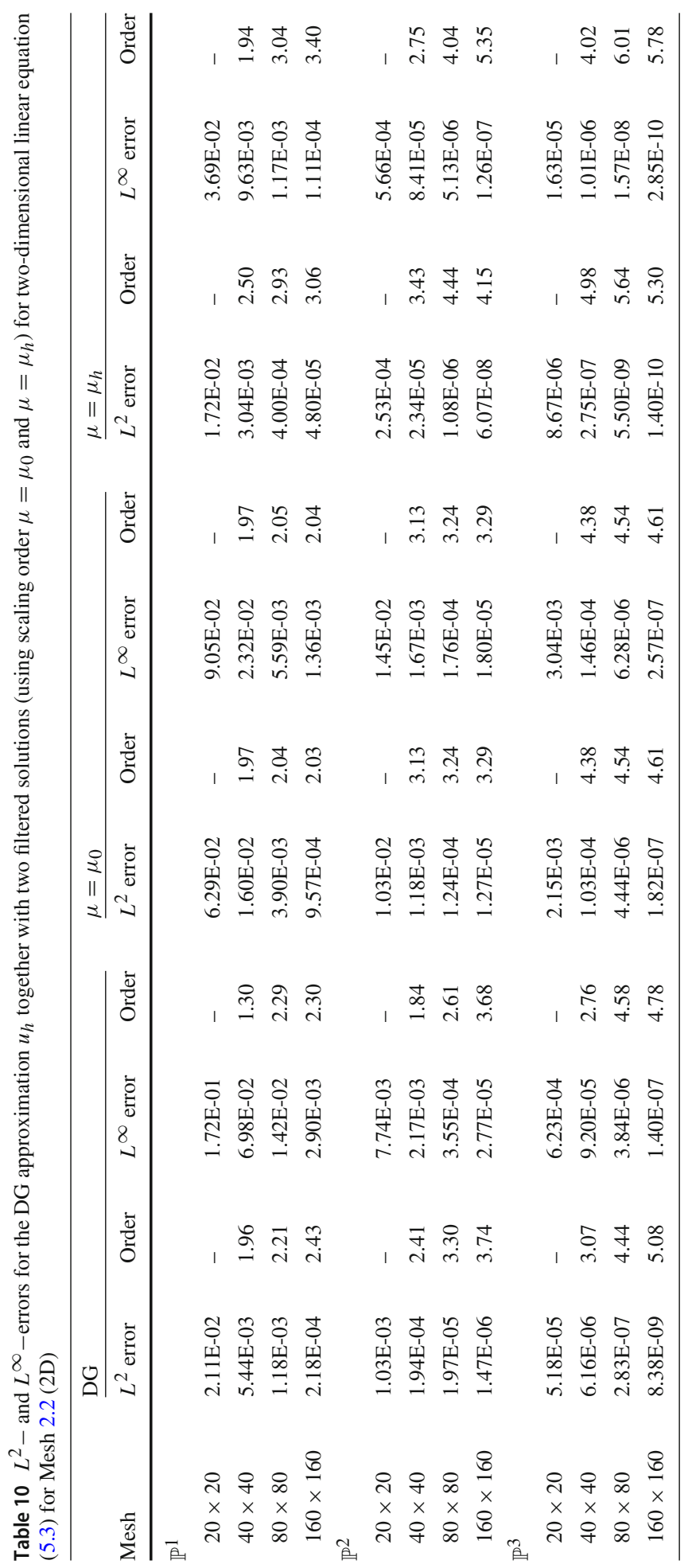



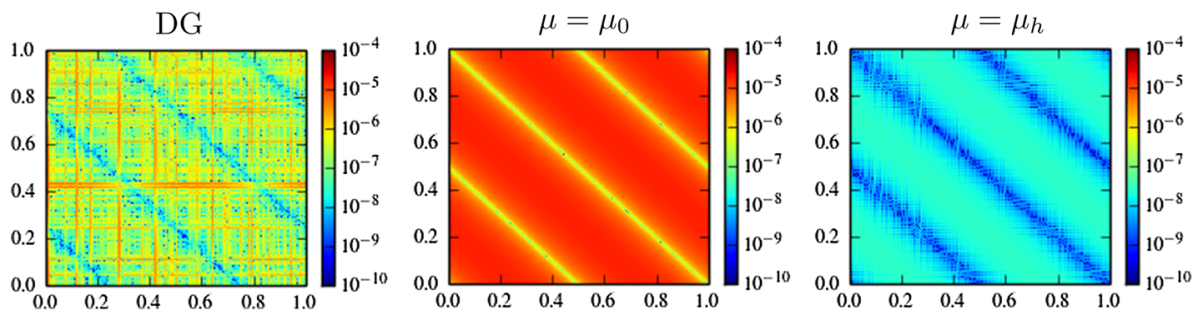

Fig. 7 Comparison of the pointwise errors in log scale of the DG approximation together with two filtered solutions (using scaling order $\mu=\mu_{0}$ and $\mu=\mu_{h}$ ) for two-dimensional linear equation (5.3) for Mesh 2.1 $\left(2 \mathrm{D}, \mathbb{P}^{2}\right.$ and $\left.N=160 \times 160\right)$
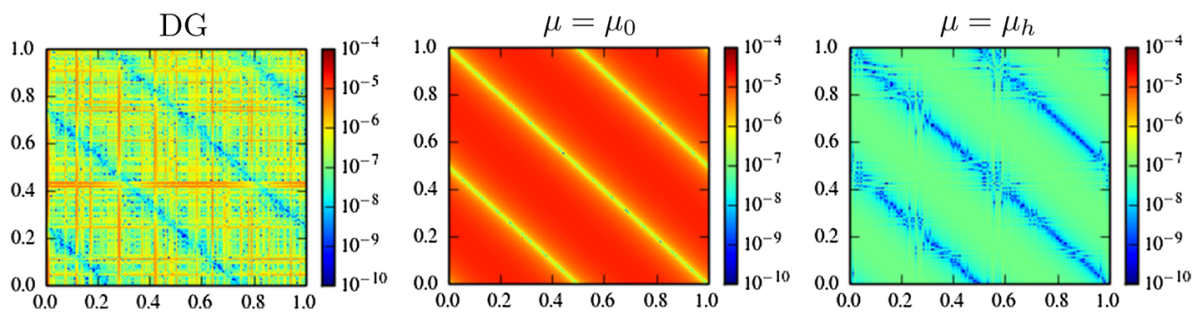

Fig. 8 Comparison of the pointwise errors in log scale of the DG approximation together with two filtered solutions (using scaling order $\mu=\mu_{0}$ and $\mu=\mu_{h}$ ) for two-dimensional linear equation (5.3) for Mesh 2.2 $\left(2 \mathrm{D}, \mathbb{P}^{2}\right.$ and $\left.N=160 \times 160\right)$

scaling order $\mu_{h}$ is chosen according to the unstructuredness of the given nonuniform meshes. Furthermore, we have numerically shown that by using the proposed scaling $H=h^{\mu_{h}}$, the filtered solutions have an accuracy order of $\mu_{h}(2 k+2)$, which is higher than the accuracy order of the DG solutions. The numerical results are promising: compared to the original DG errors, the filtered error scaling order $\mu_{h}$ has a significantly reduced error from the original DG solution as well as increased accuracy order. Also, a scaling performance validation based on a large number of nonuniform meshes has demonstrated the superiority of our proposed scaling compared to other existing methods. Future work will concentrate on extending this scaling order $\mu_{h}$ to unstructured triangular meshes in two dimensions and tetrahedral meshes in three dimensions.

Acknowledgements This work was performed while the first author was sponsored by the National Natural Science Foundation of China (NSFC) under Grants No. 11801062 and the Fundamental Research Funds for the Central Universities. The first and second authors were sponsored by the Air Force Office of Scientific Research (AFOSR), Air Force Material Command, USAF, under Grant Number FA8655-09-1-3017. The third author was sponsored in part by the Air Force Office of Scientific Research (AFOSR), Computational Mathematics Program, under Grant Number FA9550-12-1-0428. The U.S Government is authorized to reproduce and distribute reprints for Governmental purposes notwithstanding any copyright notation thereon.

Open Access This article is distributed under the terms of the Creative Commons Attribution 4.0 International License (http://creativecommons.org/licenses/by/4.0/), which permits unrestricted use, distribution, and reproduction in any medium, provided you give appropriate credit to the original author(s) and the source, provide a link to the Creative Commons license, and indicate if changes were made. 


\section{References}

1. Bramble, J.H., Schatz, A.H.: Higher order local accuracy by averaging in the finite element method. Math. Comp. 31(137), 94-111 (1977)

2. Cockburn, B.: An introduction to the discontinuous Galerkin method for convection-dominated problems. In: Quarteroni, A. (ed.) Advanced Numerical Approximation of Nonlinear Hyperbolic Equations. Lecture Notes in Mathematics, vol. 1697. Springer, Berlin, Heidelberg (1998)

3. Cockburn, B., Luskin, M., Shu, C.-W., Süli, E.: Enhanced accuracy by post-processing for finite element methods for hyperbolic equations. Math. Comput. 72(242), 577-606 (2003)

4. Cockburn, B., Shu, C.-W.: Runge-Kutta discontinuous Galerkin methods for convection-dominated problems. J. Sci. Comput. 16(3), 173-261 (2001)

5. Curtis, S., Kirby, R.M., Ryan, J.K., Shu, C.-W.: Postprocessing for the discontinuous Galerkin method over nonuniform meshes. SIAM J. Sci. Comput. 30(1):272-289, (2007/08)

6. Docampo-Sánchez, J., Ryan, J.K., Mirzargar, M., Kirby, R.M.: Multi-dimensional filtering: reducing the dimension through rotation. SIAM J. Sci. Comput. 39(5), A2179-A2200 (2017)

7. Gottlieb, S., Shu, C.W.: Total variation diminishing Runge-Kutta schemes. Math. Comput. 67(221), 73-85 (1998)

8. Hirsch, C.: Numerical Computation of Internal and External Flows: The Fundamentals of Computational Fluid Dynamics. Butterworth-Heinemann, Oxford (2007)

9. Jallepalli, A., Docampo-Sánchez, J., Ryan, J.K., Haimes, R., Kirby, R.M.: On the treatment of field quantities and elemental continuity in FEM solutions. IEEE Trans. Vis. Comput. Graph. 24(1), 903-912 (2018)

10. Ji, L., van Slingerland, P., Ryan, J.K., Vuik, K.: Superconvergent error estimates for position-dependent smoothness-increasing accuracy-conserving (SIAC) post-processing of discontinuous Galerkin solutions. Math. Comput. 83(289), 2239-2262 (2014)

11. Li, X., Ryan, J.K., Kirby, R.M., Vuik, C.: Smoothness-Increasing Accuracy-Conserving (SIAC) filters for derivative approximations of discontinuous Galerking (DG) solutions over nonuniform meshes and near boundaries. J. Comput. Appl. Math. 294, 275-296 (2016)

12. Mirzaee, H., King, J., Ryan, J.K., Kirby, R.M.: Smoothness-Increasing Accuracy-Conserving filters for discontinuous Galerkin solutions over unstructured triangular meshes. SIAM J. Sci. Comput. 35(1), A212-A230 (2013)

13. Mirzargar, M., Jallepalli, A., Ryan, J.K., Kirby, R.M.: Hexagonal Smoothness-Increasing AccuracyConserving filtering. J. Sci. Comput. 73(2), 1072-1093 (2017)

14. Mock, Michael S., Lax, Peter D.: The computation of discontinuous solutions of linear hyperbolic equations. Commun. Pure Appl. Math. 31(4), 423-430 (1978)

15. Nguyen, D., Peters, J.: Nonuniform discontinuous Galerkin filters via shift and scale. SIAM J. Numer. Anal. 54(3), 1401-1422 (2016)

16. Peters, J.: General spline filters for discontinuous Galerkin solutions. Comput. Math. Appl. 70(5), 10461050 (2015)

17. Ryan, J.K.: Local Derivative Post-processing: Challenges for a Non-uniform Mesh. Delft University of Technology Report 10-18, (2013)

18. Ryan, J.K., Cockburn, B.: Local derivative post-processing for the discontinuous Galerkin method. J. Comput. Phys. 228(23), 8642-8664 (2009)

19. Ryan, J.K., Shu, C.-W.: On a one-sided post-processing technique for the discontinuous Galerkin methods. Methods Appl. Anal. 10(2), 295-307 (2003)

20. Ryan, J.K., Li, X., Kirby, R.M., Vuik, K.: One-sided position-dependent Smoothness-Increasing Accuracy-Conserving (SIAC) over uniform and non-uniform meshes. J. Sci. Comput. 64(3), 773-817 (2015)

21. Steffen, M., Curtis, S., Kirby, R.M., Ryan, J.K.: Investigation of Smoothness-Increasing AccuracyConserving filters for improving streamline integration through discontinuous fields. IEEE Trans. Vis. Comput. Graph. 14(3), 680-692 (2008)

22. Thomée, V.: High order local approximations to derivatives in the finite element method. Math. Comput. 31(139), 652-660 (1977)

23. van Slingerland P., Ryan, J.K., Vuik, K. Smoothness-Increasing Convergence-Conserving Spline Filters Applied to Streamline Visualization of DG Approximations. Delft University of Technology Report 09-06, (2009)

24. van Slingerland, P., Ryan, J.K., Vuik, K.: Position-dependent smoothness-increasing accuracy-conserving (SIAC) filtering for improving discontinuous Galerkin solutions. SIAM J. Sci. Comput. 33(2), 802-825 (2011) 
Publisher's Note Springer Nature remains neutral with regard to jurisdictional claims in published maps and institutional affiliations.

\section{Affiliations}

\section{Xiaozhou $\mathrm{Li}^{1}$ - Jennifer K. Ryan ${ }^{2,3} \cdot$ Robert M. Kirby ${ }^{4} \cdot$ Kees Vuik ${ }^{5}$}

$凶 \quad$ Jennifer K. Ryan

Jennifer.Ryan@uea.ac.uk; Jennifer.Ryan@hhu.de

Xiaozhou Li

xiaozhouli@uestc.edu.cn

Robert M. Kirby

kirby@cs.utah.edu

Kees Vuik

C.Vuik@tudelft.nl

1 School of Mathematical Sciences, University of Electronic Science and Technology of China, Chengdu, China

2 School of Mathematics, University of East Anglia, Norwich, UK

3 Mathematics Institute, Heinrich-Heine University, Düsseldorf, Germany

4 School of Computing, University of Utah, Salt Lake City, Utah, USA

5 Delft Institute of Applied Mathematics, Delft University of Technology, 2628 CD Delft, The Netherlands 